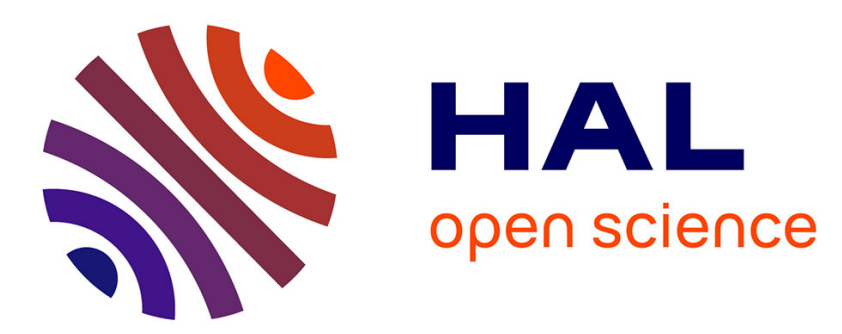

\title{
Planetary waves in a coupled chemistry-climate model: analysis techniques and comparison with reanalysis data
}

F. Mager, M. Dameris

\section{To cite this version:}

F. Mager, M. Dameris. Planetary waves in a coupled chemistry-climate model: analysis techniques and comparison with reanalysis data. Atmospheric Chemistry and Physics Discussions, 2005, 5 (3), pp.2559-2598. hal-00303904

\section{HAL Id: hal-00303904 https://hal.science/hal-00303904}

Submitted on 3 May 2005

HAL is a multi-disciplinary open access archive for the deposit and dissemination of scientific research documents, whether they are published or not. The documents may come from teaching and research institutions in France or abroad, or from public or private research centers.
L'archive ouverte pluridisciplinaire HAL, est destinée au dépôt et à la diffusion de documents scientifiques de niveau recherche, publiés ou non, émanant des établissements d'enseignement et de recherche français ou étrangers, des laboratoires publics ou privés. 
Planetary waves in model and reanalysis

\section{Planetary waves in a coupled} chemistry-climate model: analysis techniques and comparison with

\section{reanalysis data}

F. Mager and M. Dameris

F. Mager ${ }^{1, *}$ and M. Dameris ${ }^{1}$

${ }^{1}$ Institut für Physik der Atmosphäre, DLR Oberpfaffenhofen, Münchner Str. 20, D-82234

Wessling, Germany

"now at: Department of Geography, University of Cambridge, Downing Place, Cambridge CB2 3EN, UK

Received: 24 January 2005 - Accepted: 17 March 2005 - Published: 3 May 2005

Correspondence to: F. Mager (fm265@cam.ac.uk)

(C) 2005 Author(s). This work is licensed under a Creative Commons License.

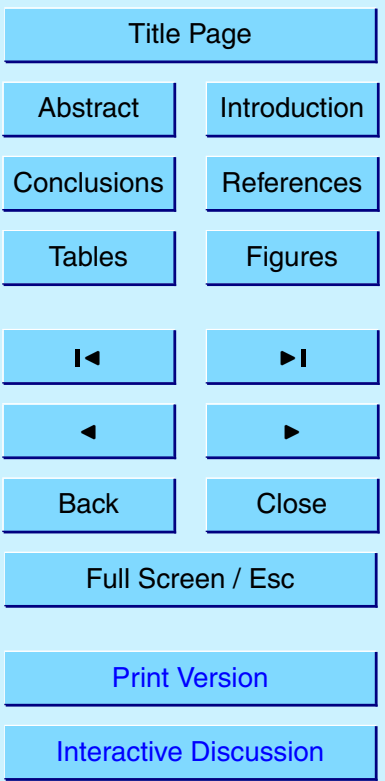

EGU 


\section{Abstract}

This paper presents several analysis techniques relating to large-scale atmospheric waves. Such analysis tools allow the extraction of planetary waves from reanalysis or model datasets, and can contribute to a detailed insight into the forcing, propagation, 5 and vertical structure of planetary waves, and their dynamic impact on the atmosphere. The different tools presented here use time series of space Fourier coefficients in order to extract transient and stationary wave parts by zonal wavenumbers, and to quantify their dynamic effect in the form of sensible heat and momentum fluxes. In this work, they have been applied to model results from the coupled chemistry-climate model ECHAM4.L39(DLR)/CHEM (E39/C) (Hein et al., 2001) and to the ERA-15 reanalysis dataset from ECMWF. We show that E39/C qualitatively matches the variance distribution and vertical structure of transient waves from reanalysis data; quantitative differences can be traced back to the horizontal model resolution and the modelled zonal winds. The modelled polar vortex during Northern Hemisphere winter has previously 15 been shown to be colder and more stable than observed (Hein et al., 2001; Schnadt et al., 2002); a possible explanation is that in the model experiment, a reduced heat flux by long transient waves at high latitudes disturbs and warms the polar vortex less than ERA-15 suggests, thereby leading to an overestimated stationary wavenumber 1 in E39/C. The results show that the tools used are well suited to investigate and estimate the impact of various dynamic processes related to large-scale waves.

\section{Introduction}

The interactions of physical, dynamical and chemical processes are manifold. Therefore, it is often difficult to assess and especially to quantify the rate at which these distinct processes are contributing to observed changes and trends in the atmosphere.

A typical question is to what extend the reduction/recovery of the ozone layer has been/will be influenced by the greenhouse effect. Since the greenhouse effect yields
ACPD

$5,2559-2598,2005$

Planetary waves in model and reanalysis

F. Mager and M. Dameris

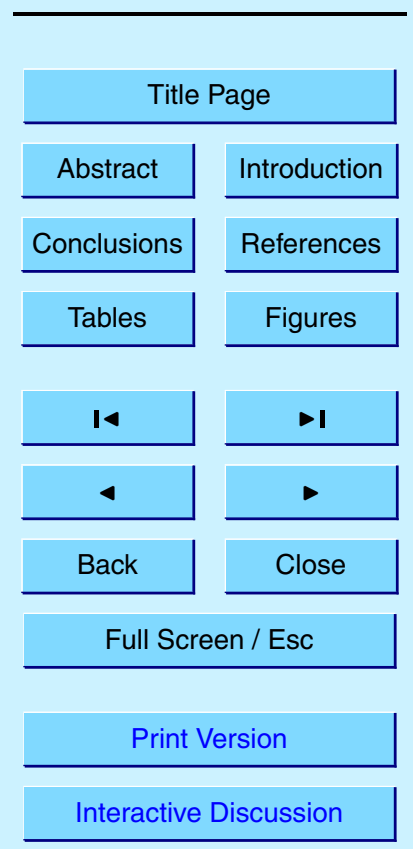

EGU 
a warming of the troposphere and a cooling of the stratosphere (e.g. IPCC, 2001; Shine et al., 2003) the answer is not trivial. Whereas the cooling of the stratosphere increases the amount of polar stratospheric clouds (PSCs) and therefore the potential of enhanced ozone reduction, the warming of the troposphere modifies the forcing and 5 upward propagation of large-scale planetary waves. Changes in the "dynamical heating" of the stratosphere can strongly impact the (dynamical and chemical) behaviour of the ozone layer (e.g. Schnadt et al., 2002).

Fully coupled chemistry-climate models (CCMs) can be used to address some of these questions. Recently, a number of CCMs have been established to simulate past, 10 present and future atmospheric conditions (e.g. Rozanov et al., 2001; Austin, 2002; Schnadt et al., 2002; Nagashima et al., 2002; Pitari et al., 2002; Steil et al., 2003). The uncertainties and assessments of currently available CCMs have been discussed in Austin et al. (2003).

There are obvious differences between the results of the employed CCMs. Several 15 deficiencies have been indicated, for example, the models did not uniformly reproduce the observed ozone changes (trends) in different latitudinal regions and were not able to simulate the observed water vapour trends in the stratosphere. An important disadvantage of most models is the "cold bias" problem, i.e., much too low temperatures simulated in the lower stratosphere, particularly at polar latitudes in winter and spring.

It was shown that most CCMs underestimate the meridional heat fluxes, which certainly has an impact on the distribution of chemical species (tracers) (e.g. Newman and Nash, 2000). This is an indication that the forcing of large-scale planetary waves, their propagation through the stratosphere and their interaction with the mean flow is not adequately represented by the models. Therefore, it is no surprise that the progno25 sis of the distinct CCMs significantly differs with regards to the recovery of the ozone layer. Particularly in the Northern Hemisphere $(\mathrm{NH})$, where dynamical processes play a dominant role, the models show large differences, indicating that future assessments are currently highly unreliable.

This paper aims to investigate the large-scale (planetary) wave activity during $\mathrm{NH}$
ACPD

5, 2559-2598, 2005

\section{Planetary waves in model and reanalysis}

F. Mager and M. Dameris

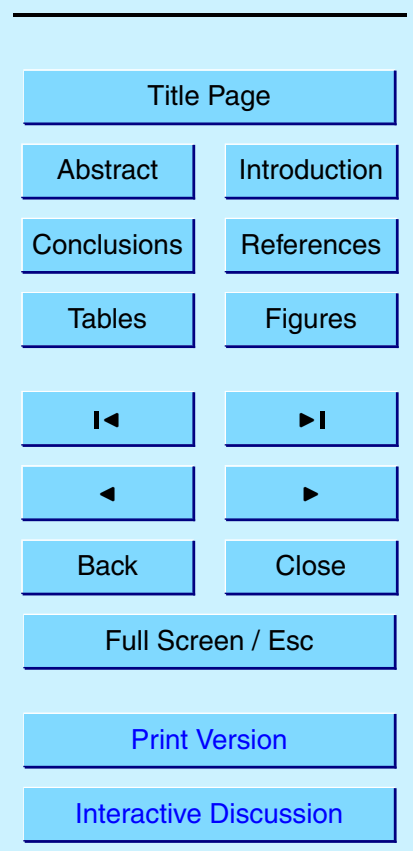

EGU 
winter (DJF) in the fully coupled chemistry-climate model ECHAM4.L39(DLR)/CHEM (E39/C). The results of a time-slice experiment, representing "1990" atmospheric conditions (Hein et al., 2001), are analysed and compared to ECMWF reanalysis data (ERA-15, 1984-1993). In particular, a wavenumber-frequency analysis (WFA) is used 5 to detect the types of planetary waves (i.e., standing and transient waves; forced and normal modes) which are in-situ generated in the model. Hayashi (1977) presented a spectral analysis applied to a given space-time series to classify atmospheric (planetary) waves. The power spectra are decomposed into standing and travelling parts, and the power of the travelling waves is partitioned into pure eastward and westwards 10 moving components (see Sects. 2). This space-time spectral analysis represents a powerful diagnostic tool to detect and to study how atmospheric waves are maintained in multi-year observations and long-term numerical model simulations, e.g., simulations with climate models over years or decades. It can ideally be employed to check the model's ability to generate a reasonable wave spectrum, which itself is the basis 15 for a realistic representation of atmospheric dynamics. In spite of this, it is a diagnostic technique that has rarely been used. An update was given by Hayashi (1982). Hayashi and coworkers themselves make use of it for several studies (e.g. Hayashi and Golder, 1977, 1983a,b, 1994; Hayashi et al., 1997), in particular for the analysis of different waves in the GFDL GCM ("SKYHI"). Speth and Kirk (1981) and Speth and Madden 20 (1983) employed the Hayashi method for space-time spectral analyses of long time series of observed geopotential heights to establish typical periods and structures of large-scale wave-like disturbances. Model data were investigated by Jakobs and Hass (1987). They used this analysis technique to show that large-scale perturbations in the mesopause region of their model correspond to normal modes. Recently, Miyoshi 25 (1999) and Miyoshi and Hirooka (1999) examined the behaviour of the 5-day and the 16-day wave in the mesopause region of their GCM (Kyushu University).

The different techniques to extract transient and stationary waves and to assess their properties are described in Sect. 2 of this paper. In the following section, the employed model E39/C is briefly presented, as well as the model and reanalysis data (ERA-15)

ACPD

$5,2559-2598,2005$

\section{Planetary waves in model and reanalysis}

F. Mager and M. Dameris

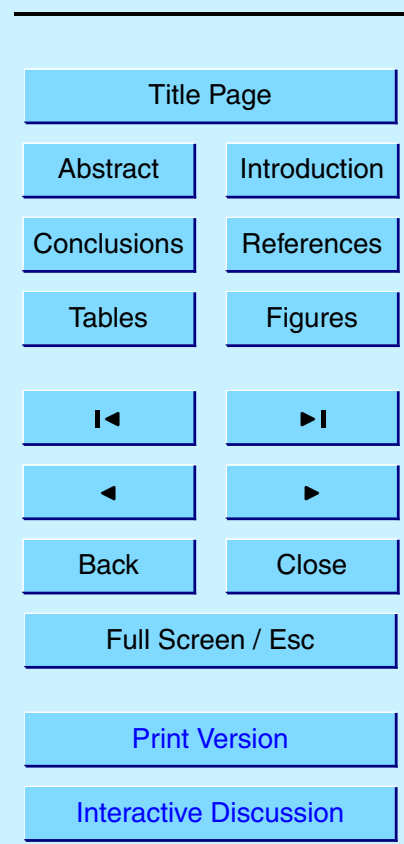

EGU 
used for the investigations. Section 4 shows a validation of E39/C that is based on the comparison of the "1990" time-slice simulation and ERA-15 data, in order to assess the model's ability to reproduce the observed wave activity and its dynamic impact. This section also tries to answer the question of why the northern polar vortex in the model 5 is colder and stabler than observed. A discussion and conclusion is given in the last section.

\section{Methodology}

\subsection{Description of the wavenumber-frequency analysis}

In this section, we will briefly describe the space-time spectral analysis after Hayashi 10 (1977, 1982). The method resolves transient waves at distinct wavenumbers into standing and eastward and westward travelling waves at different frequencies and, additionally, computes the coherence and phase difference between two time series. Such a wavenumber-frequency analysis (WFA) can be performed by using power spectra, cospectra and quadrature spectra of the time series to be considered. The spectra

have to be derived by time spectral analysis methods such as the maximum entropy method, the direct Fourier transform method or the lag correlation method. We will use the lag correlation method in the following to compute the mentioned spectra.

For this analysis, transient waves are assumed to propagate along latitude circles $(W=W(x, t))$. The respective time series of space-Fourier coefficients have to be extracted or computed from observational, reanalysis or model datasets for discrete zonal wavenumbers $k$ and on latitude circles.

ACPD

$5,2559-2598,2005$

Planetary waves in model and reanalysis

F. Mager and M. Dameris

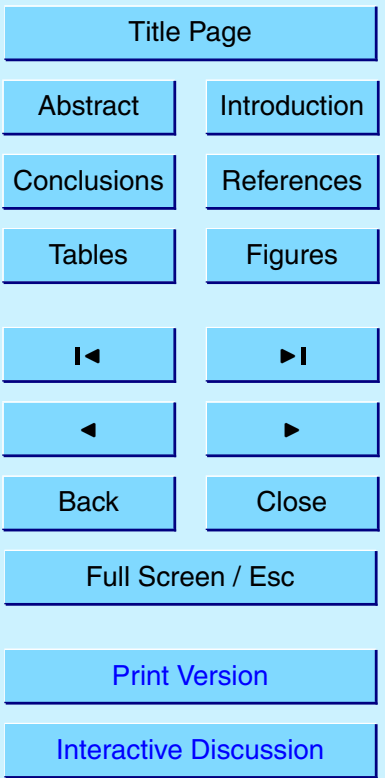

EGU 
For two real Fourier coefficient time series $x$ and $y$, spectral analysis defines the autocovariance $C V_{x x}$ and crosscovariance $C V_{x y}$ as

$C V_{x x}(\tau)=\lim _{T \rightarrow \infty} \frac{1}{2 T} \int_{-T}^{T} x(t) x(t+\tau) d t$

$C V_{x y}(\tau)=\lim _{T \rightarrow \infty} \frac{1}{2 T} \int_{-T}^{T} x(t) y(t+\tau) d t$

where $T$ and $\tau$ denote the length of the time series and a chosen lag, respectively.

In reality, time series will be limited in time, and spectral values will not be calculated for single frequencies $\omega$ but for frequency intervals. The interval width $\Delta \omega=\left(2 \tau_{\text {max }}\right)^{-1}$ depends on the chosen maximal lag $\tau_{\max }$ which should not exceed one-third of the time series length. For convenience, we will use $\omega$ instead of $\Delta \omega$ in the following equations.

The Fourier transform of the autocovariance function

$P_{X X}(\omega)=\frac{1}{2 \pi} \int_{-\infty}^{\infty} C V_{x X}(\tau) e^{-i \omega \tau} d \tau$

and its symmetry $C V_{x x}(\tau)=C V_{x x}(-\tau)$ lead to the real power spectrum

$P_{x}(\omega):=P_{x x}(\omega)=\frac{1}{2 \pi} \int_{-\infty}^{\infty} C V_{x x}(\tau) \cos (\omega \tau) d \tau$

Planetary waves in model and reanalysis

F. Mager and M. Dameris

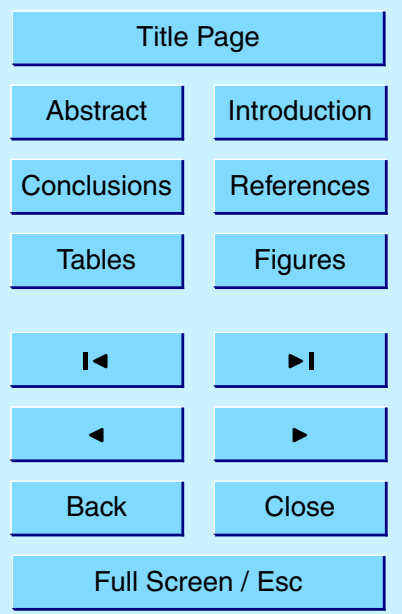

Print Version

Interactive Discussion

EGU 
which contains the variance per circular frequency $\omega$. The Fourier transform of the crosscovariance yields the cospectrum $K_{x y}$ and the quadrature spectrum $Q_{x y}$

$K_{x y}(\omega)=R e\left(P_{x y}(\omega)\right)=\frac{1}{2 \pi} \int_{-\infty}^{\infty} C V_{x y}(\tau) \cos (\omega \tau) d \tau$

$Q_{x y}(\omega)=-I m\left(P_{x y}(\omega)\right)=\frac{1}{2 \pi} \int_{-\infty}^{\infty} C V_{x y}(\tau) \sin (\omega \tau) d \tau$

5 where $K_{x y}$ describes whether two oscillations propagate with equal or opposite phase; $Q_{x y}$ contains information about the phase difference between two time series.

2.1.2. Transient wave analysis after Hayashi

In order to separate standing waves from eastward and westward travelling waves, Hayashi $(1977,1982)$ proposed a method based on the following assumptions:

a) Standing waves consist of eastward and westward moving coherent components of equal amplitudes.

b) Travelling waves consist of eastward and westward moving incoherent components.

c) Standing and travelling waves are incoherent to each other and of different origin.

15 The total variance of a time series, including standing as well as eastward and westward travelling waves, is given by

\section{$5,2559-2598,2005$}

Planetary waves in model and reanalysis

F. Mager and M. Dameris

$P_{\text {tot }}(k, \pm \omega)=\frac{1}{4}\left[P_{a_{k}}(\omega)+P_{b_{k}}(\omega) \pm 2 Q_{a_{k} b_{k}}(\omega)\right]$

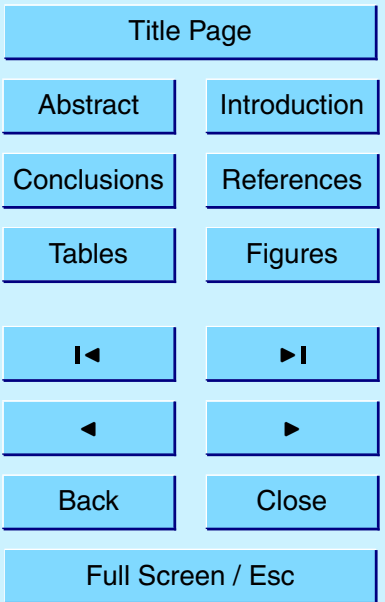

Print Version

Interactive Discussion

EGU 
where $k$ is an integer wavenumber and $a_{k}$ and $b_{k}$ are the cosine/sine Fourier coeffiACPD cient time series. $P_{a_{k}}$ and $P_{b_{k}}$ denote their power spectra, $Q_{a_{k} b_{k}}$ is the quadrature spectrum (see Sect. 2.1.1). Using the assumptions mentioned above, Hayashi deduced the standing part

$P_{s t}(k, \omega)=\sqrt{\frac{1}{4}\left(P_{a_{k}}(\omega)-P_{b_{k}}(\omega)\right)^{2}+K_{a_{k} b_{k}}^{2}(\omega)}$

and the purely eastward or westward travelling parts $( \pm \omega)$

$P_{\text {prog }}(k, \pm \omega)=P_{\text {tot }}(k, \pm \omega)-\frac{1}{2} P_{s t}(k,|\omega|)$.

As stated above in assumption a), standing waves are formed by eastward and westward travelling waves that are coherent with each other. Therefore, the variance of standing wave parts is noise-free, contrary to travelling parts, because the noise component results from travelling wave parts which are incoherent with each other (see Fig. 1).

The cospectrum $K^{R \varphi}$ and the quadrature spectrum $Q^{R \varphi}$

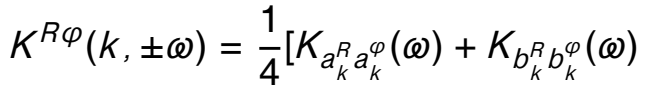

$$
\begin{aligned}
& \left. \pm Q_{a_{k}^{R} b_{k}^{\varphi}}(\omega) \mp Q_{b_{k}^{R} a_{k}^{\varphi}}(\omega)\right]
\end{aligned}
$$

$$
\begin{aligned}
Q^{R \varphi}(k, \pm \omega)= & \frac{1}{4}\left[K_{b_{k}^{R} a_{k}^{\varphi}}(\omega)-K_{a_{k}^{R} b_{k}^{\varphi}(\omega)}\right. \\
& \pm Q_{a_{k}^{R} a_{k}^{\varphi}}(\omega) \pm Q_{\left.b_{k}^{R} b_{k}^{\varphi}(\omega)\right]}
\end{aligned}
$$

allow the coherence $\operatorname{Coh}(k, \pm \omega)$ and phase difference $\Delta P h(k, \pm \omega)$

$\operatorname{Coh}^{2}(k, \pm \omega)=\frac{\left[K^{R \varphi}(k, \pm \omega)\right]^{2}+\left[Q^{R \varphi}(k, \pm \omega)\right]^{2}}{P_{t o t}^{R}(k, \pm \omega) \cdot P_{t o t}^{\varphi}(k, \pm \omega)}$

\section{Planetary waves in model and reanalysis}

F. Mager and M. Dameris

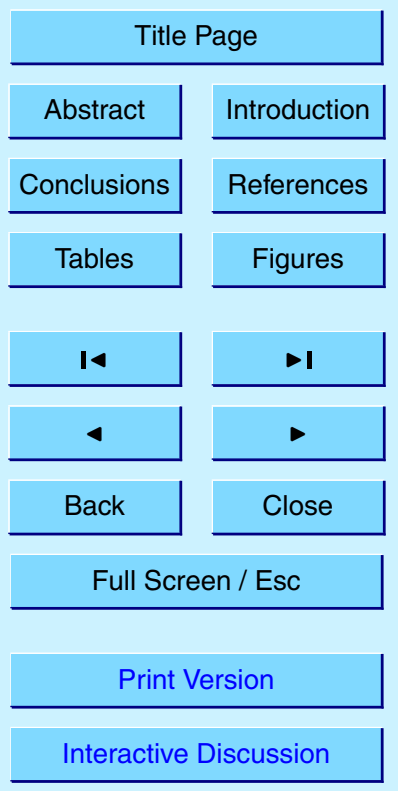

EGU 
$\Delta P h(k, \pm \omega)=\tan ^{-1} \frac{Q^{R \varphi}(k, \pm \omega)}{K^{R \varphi}(k, \pm \omega)}$

ACPD

to be computed for some reference time series $R$ and a secondary time series $\varphi$, e.g. when comparing waves on two latitude circles or two pressure levels.

5 2.1.3. Properties of transient waves

After Hartmann (1994), the mean meridional heat flux can be approximated by

$\left[\overline{v^{\prime} T^{\prime}}\right] \approx \frac{g^{2} A_{0}^{2} k p_{0}}{2 f R \Delta p} \sin \delta$

where $g, A_{0}, k$ and $p_{0}$ denote gravity, wave amplitude of geopotential height, wavenumber and pressure at a distinct pressure level; $f, R, \Delta p$ and $\delta$ denote the Coriolis parameter, gas constant, pressure difference and phase difference with respect to a higher pressure level. A baroclinic wave with a vertical tilt to the west will result in a positive $\sin \delta$ and hence in a positive heat flux towards the polar regions. Barotropic (or external) modes tend to have little or no phase tilt and therefore contribute very little to the meridional heat flux.

15 The amplitude $A$ of a barotropic wave in an isothermal atmosphere increases exponentially with height:

$A(p)=A\left(p_{0}\right) \exp \left[\frac{R}{c_{p}} \ln \frac{p_{0}}{p}\right]$

where $p_{0}, p$ and $c_{p}$ denote pressure at some starting level 0 , pressure at a higher pressure level and the specific heat of air. If the tropospheric amplitude increase of a certain wave is greater than that of the corresponding exponentially growing mode, then conversion from available potential to kinetic energy must take place, as is the case for baroclinic waves (Speth and Madden, 1983). These waves will often show

Planetary waves in model and reanalysis

F. Mager and M. Dameris

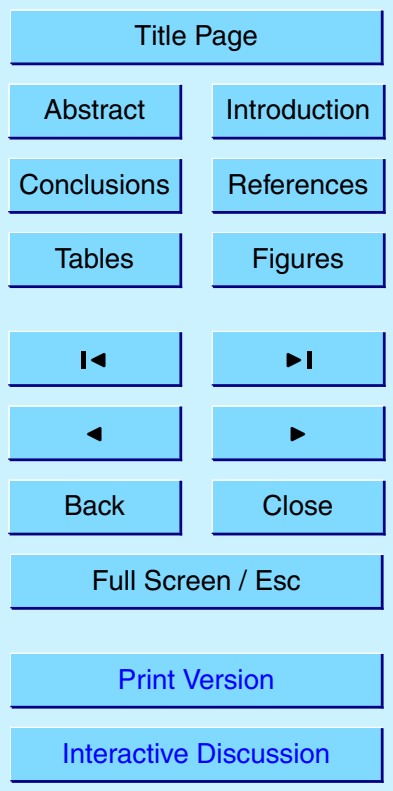

EGU 
a stratospheric amplitude decrease, because they derive a large part of their energy from the tropospheric mean flow at frontal zones (Salby, 1982).

ACPD

5, 2559-2598, 2005

\subsection{Stationary waves}

Stationary waves with an integer zonal wavenumber $k$ can be easily computed from 5 space-Fourier coefficients on latitude circles. For any particular time, vertical level and latitude, the contribution of wavenumber $k$ to some variable as temperature or geopotential height on a latitude circle with logitudinal coordinate $x$ is given by

$W_{k}(x)=2\left(a_{k} \cos k x-b_{k} \sin k x\right)$.

Hence, the sum of all $W_{k}, k=0, \ldots, k_{\max }$ results in the chosen variable field, with $k_{\max }$ 10 depending on the field's resolution. When $W_{k}$ is averaged over time, then patterns of stationary waves emerge and can be interpreted.

It should be noted that the factor and sign of the terms in Eq. (16) can differ from those used here (2 and -2$)$, depending on the definition of Fourier coefficients in the respective GCM or reanalysis scheme.

15 The troposphere and the stratosphere are dynamically linked through the vertical propagation of planetary waves. The direction and the magnitude of the zonal wind are the main factors deciding whether vertically propagating waves are dampened or not (Charney and Drazin, 1961). The so-called refractive index (Matsuno, 1970) is a useful tool in this context, as it helps to identify those regions of the atmosphere where planetary waves can propagate vertically $\left(Q_{k}\right.$ positive and not too large):

$Q_{k}(y, z)=\frac{\bar{P}_{\phi}}{\bar{u}}-\left(\frac{k}{\cos \phi}\right)^{2}-\left(\frac{a f}{2 N H}\right)^{2}$,

where $\bar{u}, k, \phi, a, f=2 \Omega \sin \phi, N$ and $H$ denote the zonal wind, zonal wavenumber, latitude, Earth radius, Coriolis parameter ( $\Omega$ angular velocity of the Earth), Brunt-Väisälä

\section{Planetary waves in model and reanalysis}

F. Mager and M. Dameris

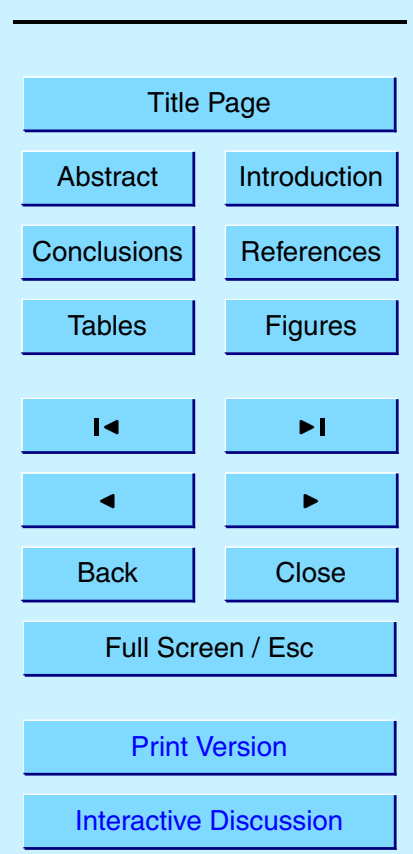

EGU 
frequency and scale height. The meridional derivative of potential vorticity is given by

$\bar{P}_{\phi}=2 \Omega a \cos \phi-\left[\frac{[\bar{u} \cos \phi]_{\phi}}{\cos \phi}\right]_{\phi}-\frac{a^{2} f^{2}}{\rho_{0}}\left[\rho_{0} \frac{\bar{u}_{z}}{N^{2}}\right]_{z}$

where []$_{\phi}$ and []$_{z}$ denote meridional and vertical partial derivatives, respectively.

It should be noted that the refractive index must be used with care, since it is a rela-

5 tively limited tool insofar as it only indicates how favourable the underlying atmospheric conditions (and especially the zonal wind) are for the transmission of waves. It cannot conclusively answer the question of how the atmosphere will exactly modify the wave behaviour, i.e. by dampening waves, by deviating them or by reflecting them back into the troposphere (Harnik and Lindzen, 2001; Perlwitz and Harnik, 2003).

\subsection{Heat and momentum fluxes}

Baroclinic planetary waves are essential for the meridional transport of eddy heat and momentum flux. A simple method to quantify the contribution of stationary and transient Rossby waves to the meridional heat flux is given by Peixoto and Oort (1992):

$[\overline{v T}]=[\bar{v}][\bar{T}]+\left[\overline{V^{*}} \overline{T^{*}}\right]+\left[\overline{V^{\prime} T^{\prime}}\right]$,

15 where the total heat flux is partitioned into the mean circulation and the contributions by stationary and transient waves (momentum fluxes can be computed analogously). This method can be further refined by computing the contributions of single wavenumbers to the transient and stationary heat flux (Newman and Nash, 2000).

\section{Model description and experimental setup}

20 In this study the interactively coupled chemistry-climate model ECHAM4.L39(DLR)/CHEM (E39/C) is used. More detailed descriptions of the 2569

\section{Planetary waves in model and reanalysis}

F. Mager and M. Dameris

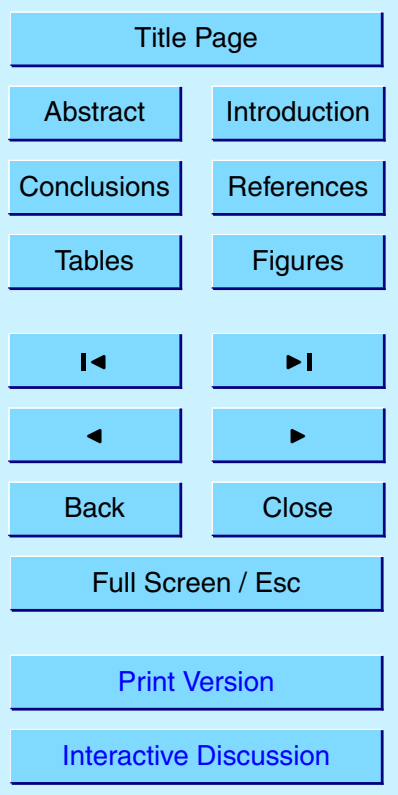

EGU 
model are given in Hein et al. (2001) and Schnadt et al. (2002). The horizontal resolution of the model is T30 $\left(3.75 \times 3.75^{\circ}\right)$. E39/C has 39 layers from the surface to the top layer centered at $10 \mathrm{hPa}$ (Land et al., 2002).

CHEM (Steil et al., 1998) is based on the family concept. It describes relevant 5 stratospheric and tropospheric $\mathrm{O}_{3}$ related homogeneous chemical reactions and heterogeneous chemistry on PSCs and sulfate aerosols. It does not consider bromine chemistry. E39/C includes online feedbacks of dynamics, chemistry, and radiative processes: chemical tracers are advected by the simulated winds. The net heating rates, in turn, are calculated using the actual 3-D distributions of the radiatively active gases $10 \mathrm{O}_{3}, \mathrm{CH}_{4}, \mathrm{~N}_{2} \mathrm{O}, \mathrm{H}_{2} \mathrm{O}$, and CFCs.

In this work, a time-slice experiment representing conditions for 1990 is evaluated. The time-slice has been integrated over 24 years under steady state conditions, with the first four years taken as spin-up. Sea surface temperatures (SST) are prescribed from observations (Gates, 1992). Additionally, natural and anthropogenic $\mathrm{NO}_{\mathrm{x}}$ emis-

15 sions at the surface, from lighthing, and by aircraft are considered. At the model top, mixing ratios of $\mathrm{NO}_{y}$ and $\mathrm{CIX}\left(=\mathrm{ClO}_{x}+\mathrm{ClONO}_{2}+\mathrm{HCl}\right)$ are prescribed to account for higher altitude chemistry above the upper boundary. Mixing ratios for the most relevant greenhouse gases $\mathrm{CH}_{4}$ and $\mathrm{N}_{2} \mathrm{O}$ (at the surface) and $\mathrm{CO}_{2}$ are specified according to observations. The specific boundary fields are given in Table 1 (Hein et al., 2001).

20 The climatology of E39/C has been extensively validated in several previous works (e.g. Hein et al., 2001; Schnadt et al., 2002; Austin et al., 2003).

\section{Data}

As stated in Sect. 2, time series of space-Fourier coefficients for integer wavenumbers of geopotential height are used to derive properties of transient and stationary waves.

25 These Fourier coefficients have been extracted from reanalysis and model data. Reanalysis data are derived from the ERA-15 ECMWF reanalysis project (Gibson et al., 1997). The original ERA-15 data have a spectral T106 resolution $\left(1.1 \times 1.1^{\circ}\right)$ with 31

ACPD

$5,2559-2598,2005$

\section{Planetary waves in model and reanalysis}

F. Mager and M. Dameris

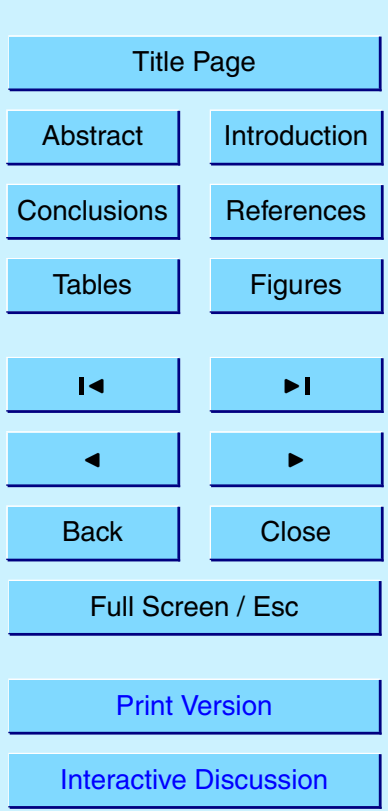

EGU 
vertical hybrid levels from the ground to an upper boundary at $10 \mathrm{hPa}$. Data exist at $0,6,12$, and 18 UTC for the period from 1979 to 1993 . These analyses of ERA-15 data were processed in order to compare them with the E39/C model data. Thus, the spectral resolution was reduced to T30 $\left(3.75 \times 3.75^{\circ}\right)$ and data were interpolated onto

517 appropriate pressure levels between 1000 and $10 \mathrm{hPa}$. As mentioned above, this study only focuses on $\mathrm{NH}$ winter (DJF). One single measurement per day was chosen at 0 UTC for DJF and the considered period was shortened by five years (1984-1993) so as to allow a comparison with the model time-slice for the year 1990. All 20 available model winters have been analysed, with space and time resolutions corresponding to 10 ERA.

\section{Results and discussion}

In this section we address the question of how accurately E39/C simulates the transient and stationary wave activity during $\mathrm{NH}$ winter. This is done by comparing modelled and reanalysed quantities which are made available by the methodology described in 15 Sect. 2: the geopotential height variance of stationary and transient waves, their distribution over frequencies and wavenumbers, their vertical structure and their dynamic impact through meridional fluxes of sensible heat. Additionally, we use the methodology to explain why the modelled polar vortex in the winter stratosphere is stronger and more stable than suggested by the reanalysis data.

20 5.1. Transient wave properties in model and reanalysis data

In the following we shall discuss the properties of transient waves that have been extracted from model and ERA-15 data.

Figure 2 displays the distribution of geopotential height variance over latitudes and zonal wavenumbers, as a sum over frequencies and directions of zonal propagation; this quantity can be considered as a rough measure for the energy of travelling waves.

ACPD

$5,2559-2598,2005$

\section{Planetary waves in model and reanalysis}

F. Mager and M. Dameris

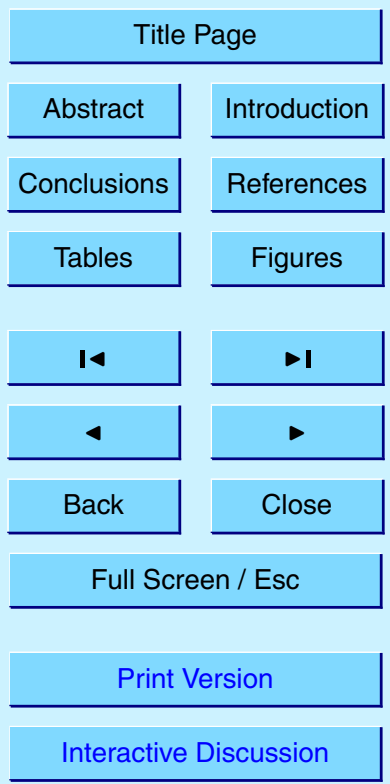

EGU 
The variances from ERA-15 data (left panel) show some well-known properties of transient waves: The largest variances can be found in the extratropics, where Rossby waves come into being through the conservation of potential vorticity. With increasing wavenumber, the meridional variance maximum tilts towards lower latitudes at all three 5 pressure levels. This should partly arise through the fact that ultra-long waves (ZWN 1 and 2) come into being at high latitudes whereas shorter waves on the synoptic scale are mostly forced at middle latitudes; another reason for this meridional distribution of wave activity may be that shorter waves have the tendency to be refracted towards regions with a higher refractive index, the index decreasing with increasing wavenum10 ber and latitude (see middle term of Eq. 17). The variances at 500 and $150 \mathrm{hPa}$ are qualitatively similar, whereas those at $50 \mathrm{hPa}$ distinctly differ: in the northern (winter) stratosphere, the variance is shifted towards small wavenumbers through the strong westerlies. These act as a low-pass filter on planetary waves (Charney and Drazin, 1961 ) by allowing waves of the smallest wavenumbers (typically 1 and 2) to prop15 agate vertically unhindered whereas waves at higher wavenumbers are dampened. In the southern (summer) hemisphere, the stratospheric variances are reduced at all wavenumbers with respect to the lowermost stratosphere $(150 \mathrm{hPa})$; here, the easterly winds (in approximate thermal wind balance with the temperature) impose a barrier on the vertical propagation of all planetary waves.

The comparison of absolute ERA and E39/C variances (left and middle panels) shows that E39/C accurately simulates the variance distribution of transient waves over latitudes and wavenumbers. The model reproduces the wave properties mentioned above, e.g. the tropical variance minimum, the equatorward variance tilt with increasing wavenumber or the dampening of waves according to their wavenumber 25 and the zonal wind. Although the model variances match the ERA-15 variances quantitatively, the variance differences (right panels) yield some valuable information about the model.

E39/C simulates approximately $25 \%$ (50\%) less tropospheric transient wave activity in the Northern (Southern) Hemisphere at all considered wavenumbers (bottom right).

ACPD

$5,2559-2598,2005$

\section{Planetary waves in model and reanalysis}

F. Mager and M. Dameris

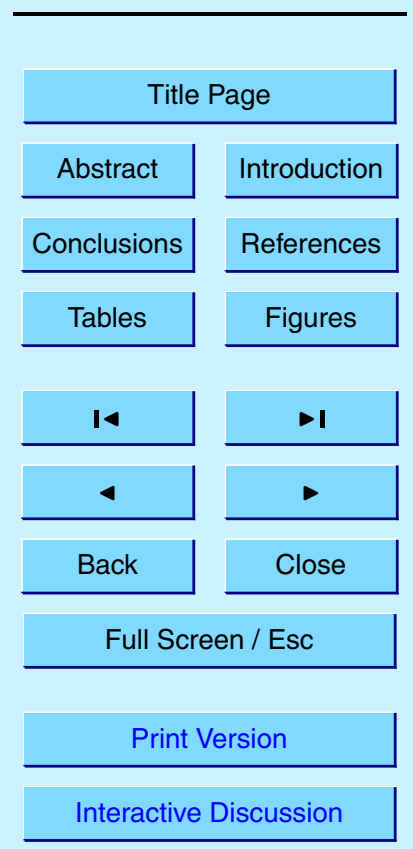

EGU 
The reason for smaller tropospheric model variances is again related to the horizontal resolution, namely the insufficient representation of cyclonic activity at resolution T30 (Senior, 1995). Although there is less forcing of tropospheric transient waves in E39/C, the modelled variances in the tropopause region at middle latitudes are higher at all 5 wavenumbers than in ERA-15 data. This is most probably due to the stronger subtropical jets in E39/C (Fig. 6, right panel) which induce higher shear instabilities and, subsequently, more transient waves in the jet region. The reduced modelled wave activity with wavenumbers 1-3 at high northern latitudes seems to arise in the troposphere and propagates with less variance into the stratosphere. The higher stratospheric model 10 variances at middle northern latitudes appear to be low-pass filtered modes that have been forced by the stronger model jet and have then propagated vertically. One of the most striking stratospheric features is, however, that E39/C allows a much larger vertical wave propagation into the summer stratosphere than ERA. This occurs because of the model's inability to correctly reproduce eastern stratospheric winds in the southern hemisphere during summer (Hein et al., 2001).

The WFA not only allows analysis of the geopotential height variance of transient waves into contributions by single wavenumbers, as done for the previous discussion, but also into variance parts by frequency intervals. Figure 3 shows how the wave activity of eastward and westward travelling waves is distributed over the considered frequency range. The $300 \mathrm{hPa}$ level has been selected because it features the largest variances of all levels (with the exception of the lower and middle stratosphere close to the northern polar vortex), and because it allows an insight into the structure of upward travelling tropospheric waves before they get partially dampened in the stratosphere. Some of the properties that have been mentioned in the discussion of Fig. 2 are obvious in the representation of ERA-15 results (left panels), e.g. that the greatest part of planetary wave activity takes place in the extratropics, or that the meridional variance maximum is displaced equatorwards with increasing wavenumber, from approximately $70^{\circ}$ (wavenumber 1 ) to $40^{\circ}$ (wavenumber 8 ).

The largest variances of westward travelling waves in the $\mathrm{NH}$ occur at wavenumber
ACPD

$5,2559-2598,2005$

\section{Planetary waves in model and reanalysis}

F. Mager and M. Dameris

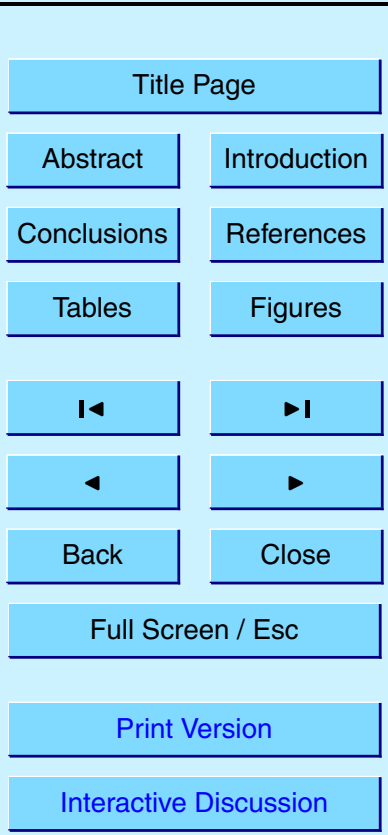

EGU 
1 and decrease monotonically with the wavenumber. In the $\mathrm{SH}$, the variances are very similar for the first three wavenumbers and then also decrease with increasing wavenumber. The strong wavenumber 1 in the $\mathrm{NH}$ appears to be the signature of the polar vortex with its stratospheric pressure dipole (aleutian high/vortex low). The $5 \mathrm{NH}$ variance of the westward travelling wave 2, being much greater than in the $\mathrm{SH}$, is probably enhanced by orographic forcing and land-sea contrast. In the southern hemisphere, the wave activity of eastward travelling waves with wavenumbers 4-7 is greater than in the $\mathrm{NH}$. The comparatively weak orography at southern middle latitudes allows higher windspeeds over the oceans and, subsequently, stronger syn10 optic disturbances at these wavenumbers which propagate vertically. With increasing wavenumber, less variance occurs in the westward direction. The transition of maxima from large westward periods ( $>20$ days at wavenumber 1 ) first to medium eastward ( 8 days at wavenumber 3 ) and then to small eastward periods (wavenumber $>3$ ) is a consequence of the dispersive nature of Rossby waves, which causes the phase 15 speed to decrease rapidly with increasing wavenumber.

The E39/C model fairly reproduces the total variance sum of transient waves quantitatively and qualitatively, as seen in Fig. 2. Hence, the model approximates the contribution of transient waves to the total kinetic energy of the atmosphere. How well does it represent the wave activity for single frequencies?

The comparison shows that the model results (right panels) are in good agreement with reanalysis variances at this particular pressure level $(300 \mathrm{hPa})$. The model correctly reproduces how meridional variance maxima are displaced equatorwards with increasing wavenumber. E39/C also accounts very accurately for the dispersion of planetary waves. Additionally, the larger $\mathrm{NH}$ amplitudes of westward travelling waves at wavenumbers 1 and 2 match those that have been derived from ERA-15. Further agreements between model and reanalysis can be seen in smaller structures. Some quantitative differences can be detected: the most prominent feature is certainly the overall smaller variances in the model results at $300 \mathrm{hPa}$. These have already been

ACPD

$5,2559-2598,2005$

\section{Planetary waves in model and reanalysis}

F. Mager and M. Dameris

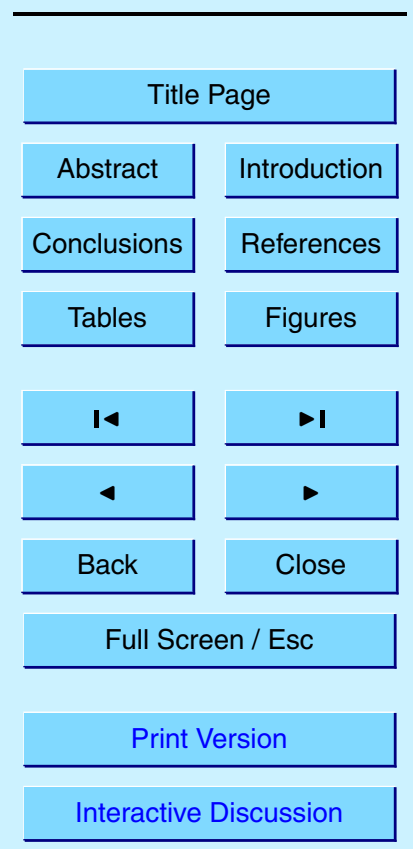

EGU 
noted in the discussion of the previous figure, where E39/C was shown to simulate less tropospheric wave activity $(500 \mathrm{hPa})$ at all considered wavenumbers. Figure 3 allows the conclusion that the model's underestimation of the tropospheric large-scale wave activity is mostly independent of wavenumber or period. Although the overall 5 pattern of modelled variance matches the reanalysis variance structures, some meridional shifts of 5 to $10^{\circ}$ occur (e.g. wavenumber 2). A few frequency shifts can also be observed, especially at the smallest wavenumbers. The patterns derived from the modelled geopotential height, despite some minor differences, are in good agreement with the results derived from reanalyses and prove that E39/C can adequately reproduce 10 the dynamic phenomena arising from transient wave formation.

Vertical profiles of wave amplitudes and phase differences are useful tools to gain an insight into the barotropic or baroclinic nature of single waves or wave groups. The amplitude can easily be derived from already computed variances under the assumption that single waves (each with a particular wavenumber in a single frequency interval) 15 perform purely harmonic oscillations; phase differences with respect to a certain pressure level can be calculated with Eq. (13).

Figure 4 shows the vertical structure of transient waves at $70^{\circ} \mathrm{N}$ (westward) resp. $50^{\circ} \mathrm{N}$ (eastward). Summing (amplitude) resp. averaging (phases) over all wavenumbers and periods can give an overview of the overall transient wave structure. In the present case, such an averaging was possible because the characteristics of individual east- and westward components were found to correspond closely to the sum resp. average.

The westward travelling wave parts from ERA-15 (solid line) show a vertical tropospheric amplitude growth which is closer to the corresponding exponential growth baroclinicity of the eastward oriented modes. This is not surprising insofar as synoptic, eastward travelling disturbances with zonal wavenumbers $>3$ are associated with baroclinic waves (Farrell, 1982). Thus, their vertical amplitude increase in the troposphere is distinctly higher than that of westward travelling wave parts. The phase differences

ACPD

5, 2559-2598, 2005

\section{Planetary waves in model and reanalysis}

F. Mager and M. Dameris

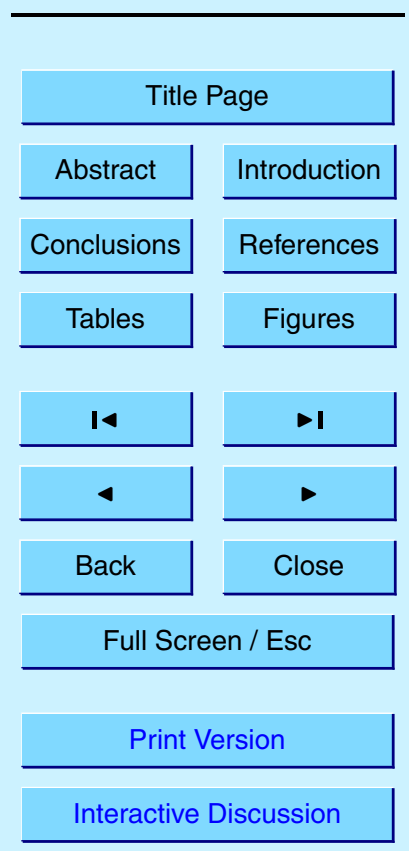

EGU 
underline this conclusion: the mean (and individual) phase difference(s) of the westward travelling waves nearly vanish(es), indicating that these modes do not significantly contribute to the meridional heat flux (Eq. 14) and are mostly barotropic. The eastward travelling wave components, on the contrary, have a very pronounced westward phase 5 tilt with height and therefore achieve a net poleward transport of sensible heat which is typical for baroclinic waves.

The comparison of the vertical structures of transient waves between ERA-15 (solid) and E39/C (dashed) at the chosen latitudes reveals that the model reproduces the ERA-15 phase differences with a high degree of similarity. The model underestimates 10 the amplitudes of westward travelling waves in the troposphere and lower stratosphere by about $10 \%$ between 850 and $70 \mathrm{hPa}$; above $70 \mathrm{hPa}$, the simulated and modelled amplitudes of these modes are nearly identical. The modelled eastward travelling wave parts match the ones from ERA-15 in the troposphere up to $300 \mathrm{hPa}$. Above this level, they are overestimated by E39/C.

15 A possible cause of the higher model amplitudes above $300 \mathrm{hPa}$ might be the height of the modelled tropopause, which lies higher than in reanalyses and observations (Santer et al., 2003). A higher tropopause could allow the model to simulate baroclinic processes higher in the troposphere than in the case of reanalysis data (roughly between 300 and $250 \mathrm{hPa}$ ), thereby inducing a higher activity of baroclinic waves with larger amplitudes. As these stronger disturbances propagate vertically, they could be the reason for the higher stratospheric wave amplitudes in the model. On the other hand, there are arguments against this interpretation which were made by Lindzen (1993). Based on theoretical assessments he found strong supporting results that the atmosphere (troposphere) tends toward baroclinic neutrality.

This analysis shows that E39/C reproduces the vertical structure of transient waves in a qualitatively satisfying way. The barotropic and baroclinic characteristics are especially well represented.

Although most atmospheric oscillations are initiated by specific causes, such as e.g. a pronounced stationary wave-2 pattern from orographic forcing, some wave
ACPD

$5,2559-2598,2005$

\section{Planetary waves in model and reanalysis}

F. Mager and M. Dameris

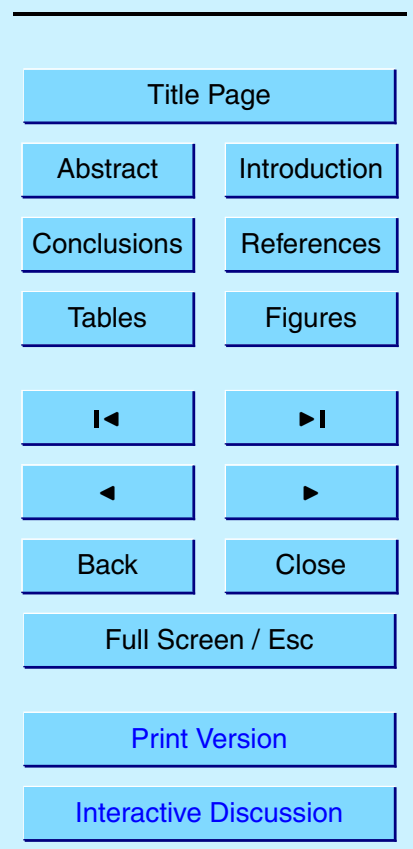

EGU 
modes can be identified which come into existence without a specific external excitation mechanism. The so-called atmospheric "normal modes" described in Sect. 2.1.3 are such oscillations. They have been predicted theoretically (Kasahara, 1976), observed (Madden, 1979) and repeatedly simulated (e.g. Miyoshi, 1999; Miyoshi and Hi5 rooka, 1999). Three observed modes exist for wavenumber 1 , with periods of 5,10 and 16 days. Figure 5 displays the corresponding periods for model and reanalysis data. These modes are barotropic waves as predicted, as they show an exponential amplitude increase with height and phase differences close to 0. ERA and E39/C normal modes have very similar amplitudes and phase differences, indicating that the model 10 possesses similar eigenvalues as observed. The methods used here are certainly not the most precise instruments to detect normal modes, but they are nevertheless useful to check the performance of climate models with regard to single wave components.

\subsection{Stationary waves and the polar vortex}

This section is devoted to the comparison of stationary waves in reanalysis and model 15 data, and especially aims to clarify why E39/C simulates a polar vortex in the time-slice "1990" which is stronger and longer-lived than in the reanalysis.

Figure 6 shows the geopotential height at $50 \mathrm{hPa}$ for $\mathrm{NH}$ winter. The modelled polar vortex is wider and deeper, and its centre is slightly displaced towards the South as compared to ERA (left, "WN0-8"). The differences are mainly due to the overestimation of stationary wavenumber 1 by E39/C (centre, "WN1"). The modelled wavenumbers 2 and 3 are weaker than in the reanalysis. Although the stronger wavenumber 1 displaces the vortex slightly southwards, weaker wavenumbers 2 and 3 imply smaller poleward heat fluxes and therefore a reduced heating of the polar region. Despite the obvious differences at all three wavenumbers, E39/C reasonably simulates the posi-

tions of extreme values as compared to ERA, with a small eastward displacement at all three wavenumbers. These differences imply that the model vortex is too strong, but that at least the position of its centre is well approximated by E39/C.

What could be the cause of the overestimated stationary wavenumber 1 as modelled 2577
ACPD

$5,2559-2598,2005$

\section{Planetary waves in model and reanalysis}

F. Mager and M. Dameris

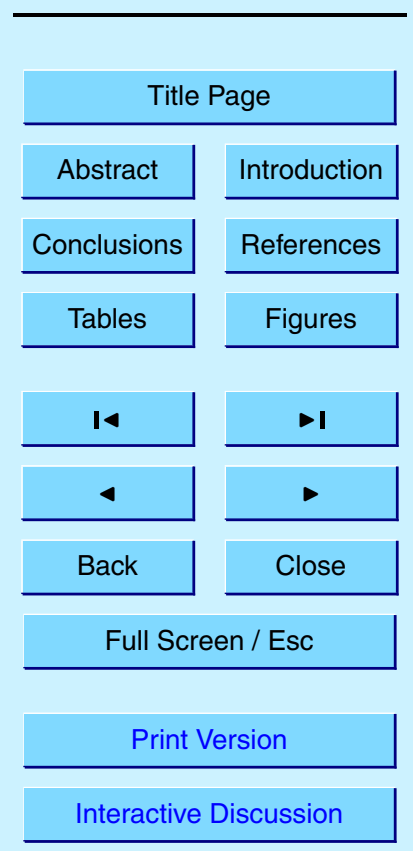

EGU 
by E39/C in DJF? Figure 7 allows the variance of stationary waves and the zonal mean zonal wind in E39/C and ERA to be compared.

The zonal wind derived from ERA (bottom left) shows the characteristic subtropical maxima at the tropopause. The $\mathrm{NH}$ jet is stronger than in the $\mathrm{SH}$ because of the 5 larger meridional temperature gradient. The southern stratosphere is dominated by easterlies, whereas westerlies prevail in the northern stratosphere. The stratospheric wind maximum around $65^{\circ} \mathrm{N}$ is the signature of the polar vortex.

Stationary waves forming in the troposphere are mainly forced by orography and land-sea contrast, which explains why the variances are larger in the $\mathrm{NH}$ and distributed 10 over a wider meridional range than in the $\mathrm{SH}$ (top left, variance over wavenumber 18). The effect of the zonal wind on the stationary wave activity is most apparent in the stratosphere, where vertically propagating waves are dampened in the $\mathrm{SH}$ by the easterly wind. In the $\mathrm{NH}$, westerlies dominate the entire extratropical stratosphere and allow stationary waves of the smallest wavenumbers (see discussion of Fig. 2) 15 to propagate vertically without any significant hindrance, thereby contributing to the characteristic shape of the northern polar vortex.

The variance of stationary modes as simulated by E39/C significantly differs from the corresponding reanalysis variance (top right). In the troposphere, the cause of the reduced resp. enhanced model variance is obvious. Here, the zonal wind difference is the dominating factor; less/more stationary waves are forced in E39/C where the modelled wind is weaker/stronger than in ERA (bottom right). The model overestimates the stationary wave variance by a factor $5-20$ in the southern middle stratosphere. This fact has already been discussed analogously for transient waves (Fig. 2), and is a consequence of the incorrect simulation of stratospheric easterly winds.

In the northern middle stratosphere, the model equally overestimates the stationary wave activity by a factor $2-5$; this signal is exclusively due to the wavenumber 1 (not shown). The representation suggests that in E39/C, too strong a zonal wind induces more tropospheric stationary wave activity between 30 and $50^{\circ} \mathrm{N}$. This signal seems to travel into the stratosphere where it is enhanced by the stronger model westerlies.

ACPD

$5,2559-2598,2005$

\section{Planetary waves in model and reanalysis}

F. Mager and M. Dameris

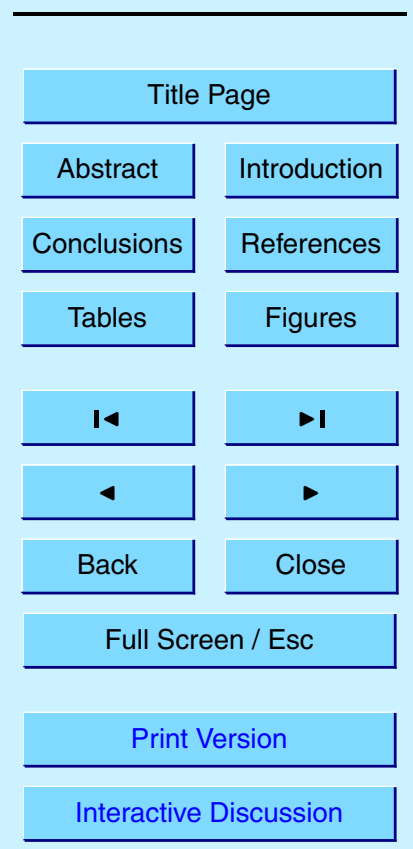

EGU 
Such a conclusion could possibly hold for the $\mathrm{SH}$ case, where the variance is strongly enhanced by a missing change of sign of the zonal wind. It does not seem to be valid for the northern stratosphere, because the model overestimates the zonal wind (westerly for E39/C and ERA) by 10-30\% only, while the variance increases by about 5 a factor 2-5; neither does this conclusion explain how such a signal between 30 and $50^{\circ} \mathrm{N}$ can spread over the whole arctic region above $100 \mathrm{hPa}$. The diffusion in the two highest model levels is increased (sponge layer) in E39/C in order to avoid reflection of waves at the upper model boundary (Hein et al., 2001), and could therefore lead to a slightly geographically wider variance distribution at these levels. However, it is 10 doubtful that this effect should vertically and meridionally affect the mentioned region to such a large extent as Fig. 7 suggests. General circulation models with sponge layers are susceptible to e.g. an imposed local force or diabatic heating inasmuch as the relaxational properties of the sponge induce changes in the dynamics outside the sponge region (Shepherd et al., 1996); however, they absorb upwelling waves realistically without causing a direct feedback on the dynamics below.

The refractive index (Eq. 17) allows us to determine those regions of the atmosphere where stationary waves with a specific wavenumber $k$ can travel vertically without being dampened. Figure 8 shows the index for ERA and E39/C for wavenumber 1, which is largely overestimated in the model simulation "1990" as discussed above. The modelled extratropical northern stratosphere is characterised by very similar, moderately positive values of the refractive index as compared to ERA and, therefore, does not obviously offer fundamentally different conditions for the vertical propagation of stationary modes with wavenumber 1 . Thus, the cause of the strong wavenumber 1 in E39/C does not appear to be primarily related to the underlying atmospheric conditions like zonal wind or vorticity. Note also the index sign in the southern extratropical stratosphere above $50 \mathrm{hPa}$; as the index is dominated by the sign of the zonal wind, it is negative for ERA and positive for E39/C, which explains why the model simulates much more stationary wave activity in the $\mathrm{SH}$ summer than the reanalysis data suggest.

Stationary waves have been shown to have larger variances in the troposphere and
ACPD

5, 2559-2598, 2005

\section{Planetary waves in model and reanalysis}

F. Mager and M. Dameris

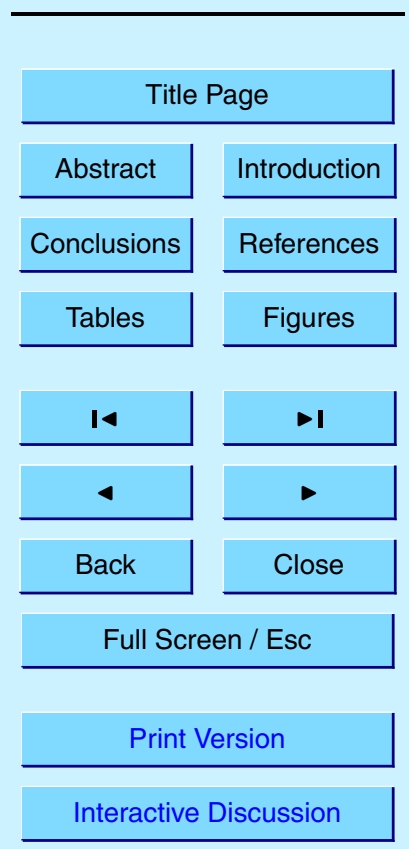

EGU 
stratosphere than transient waves at the smallest wavenumbers (1-3). The variance itself can give indications of the dynamic properties of specific wave modes, but does not allow us to quantify their dynamic impact. The meridional fluxes of momentum and sensible heat by planetary waves can be useful in this respect (Sect. 2.3), and provide 5 a plausible explanation of the stratospheric wavenumber 1 bias in E39/C.

Figure 9 shows a comparison of the variance difference (model-reanalysis) of eastward travelling waves with zonal wavenumber 1-3 to the heat flux difference by transient waves with the same wavenumbers. The comparison of meridional heat fluxes by transient and stationary waves in E39/C and ERA reveals that the model approximates 10 the reanalysis heat flux by stationary waves rather well (not shown). In contrast to this, the model clearly underestimates the $\mathrm{NH}$ heat flux by transient waves in the upper troposphere and stratosphere at high latitudes (right panel). Nearly the total heat flux difference $(>95 \%)$ is due to the wavenumbers $1-3$, which are the dominant wavenumbers at these latitudes. The vertical wave structure analysis (Sect. 5.1) has shown that the eastward travelling modes, in both model simulation and reanalysis, are predominantly baroclinic, contrary to the westward travelling wave parts. This means that most of the reduced model heat flux is likely to arise from an underestimation of eastward travelling modes. This conclusion is supported by the fact that in comparison to ERA, the tropospheric and stratospheric model variance of these modes at wavenumbers $20 \quad 1-3$ is reduced by $30-50 \%$ at high latitudes (left panel).

These results suggest the following interpretation: at high northern latitudes, E39/C simulates less eastward travelling, baroclinic waves in the troposphere than seen in the reanalysis data. These ultralong modes propagate into the stratosphere (Hartmann, 1979; Randel, 1988) and induce a weaker meridional heat flux than in ERA-15, by introducing less wave disturbances into the vortex region. If the vortex is less disturbed by a reduced heat flux, then it can cool more efficiently, leading to lower temperatures and lower pressure inside the vortex, and stronger circumpolar winds. Thus, the modelled northern polar vortex, being dominated by a quasi-permanent wavenumber 1 , exhibits a stronger stationary wavenumber 1 pattern than in the reanalysis data. This
ACPD

$5,2559-2598,2005$

\section{Planetary waves in model and reanalysis}

F. Mager and M. Dameris

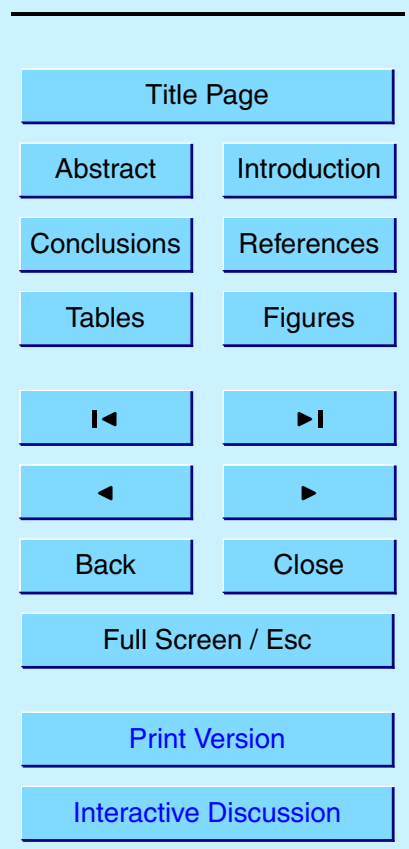

EGU 


\section{Conclusions}

In this work, we have investigated the ability of the coupled CCM E39/C to simulate 5 planetary waves. The methodology that has been used allows a detailed insight in the forcing, propagation and dynamic effect of these long atmospheric modes. The different analysis methods all use time series of space-Fourier coefficients from model simulations, observational or reanalysis data; the derived quantities provide the possibility to categorise planetary waves according to specific criteria, e.g. barotropic/baroclinic modes, transient/stationary wave parts, or their distribution over wavenumbers and frequencies. Additionally, meridional fluxes of momentum and sensible heat can be derived.

The analysis tools have been applied to a E39/C model simulation which corresponds to boundary conditions for the year "1990", as well as to the ERA-15 reanalysis data set from the ECMWF.

The comparison reveals that E39/C reproduces the qualitative distribution of geopotential height variance over wavenumbers, periods, latitudes and pressure levels in a fairly accurate way. However, too little transient wave forcing takes place in the model troposphere, which is mainly due to the underrepresentation of cyclonic activity. E39/C realistically simulates the vertical structure of transient waves, and possesses single wave modes that correspond to theoretically predicted and observed natural oscillations of the atmosphere.

Regarding the simulation of stationary waves, it has been shown that the forcing and propagation of this wave type is determined by the strength and sign of the zonal wind, especially in the troposphere. E39/C exhibits a polar vortex too strong and cold, with an overestimated stationary wavenumber 1 in the northern stratosphere. The cause appears to be an interaction of long, eastward travelling waves at high northern

\section{Planetary waves in model and reanalysis}

F. Mager and M. Dameris

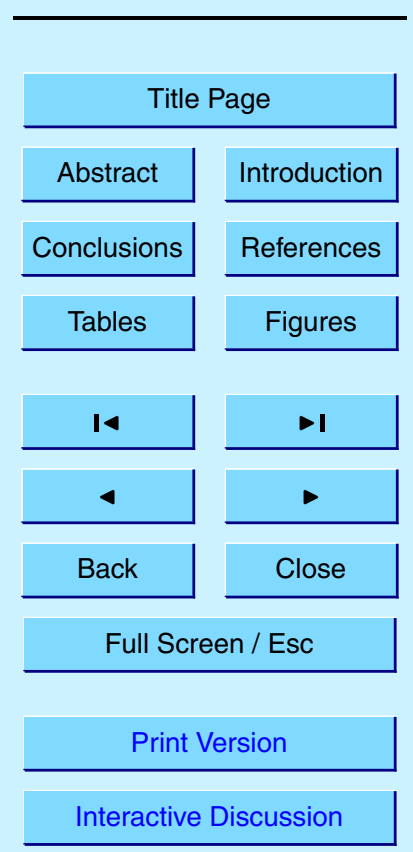

EGU 
latitudes with the stationary wavenumber 1 , linked through the meridional transport of sensible heat.

The different methods that we have presented and applied here are not fundamentally new, and have previously been used to analyse planetary wave properties in ob5 servational data as well as in simple and more complex models. However, the methodology has been employed for the first time to verify how a coupled chemistry-climate model simulates these large-scale waves both qualitatively and quantitatively. It has been demonstrated with this work that the different tools are useful in identifying and quantifying some important dynamic mechanisms which relate to planetary waves in a 10 coupled CCM. The results allow us to draw conclusions about possible model improvements that could contribute to more realistic dynamics.

The crucial factor for the correct representation of the vertical propagation of transient and stationary planetary waves in large-scale models is an accurately modelled zonal wind. Several inconsistencies of the zonal wind in E39/C have been identi-

15 fied and can be attributed to different physical causes. Thus, the subtropical jets at the tropopause are too strong in both hemispheres and, therefore, lead to more transient wave modes. The primary cause of this wind bias is an underestimation of high and middle latitude tropopause temperatures (cold bias) and a slight overestimation of equatorial temperatures in the upper troposphere (probably due to a rather rudimentary parametrisation of small-scale convective processes, see Hein et al., 2001). The inaccurately modelled easterlies in the southern stratosphere affect transient and stationary wave propagation, and most probably originate as well from the unrealistic temperature distribution at high latitudes (cold bias). The modelled stratospheric polar vortex in the high northern latitudes is characterised by zonal winds that are stronger than in ERA-15, which seems to be indirectly due to a reduced heat flux from eastward travelling transient waves.

A higher model boundary would allow for wave reflection in the middle stratosphere (Harnik and Lindzen, 2001; Perlwitz and Harnik, 2003). Using a higher horizontal model resolution (e.g. T63) would certainly contribute to a more realistic representation of

ACPD

5, 2559-2598, 2005

\section{Planetary waves in model and reanalysis}

F. Mager and M. Dameris

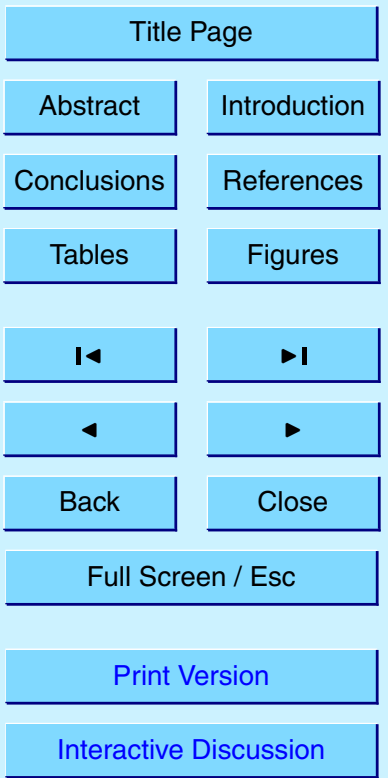

EGU 
cyclonic activity at middle latitudes, thereby improving the modelled transient wave activity. Additionally, an increased horizontal resolution would imply a less idealised orography; this should improve the orographic forcing of stationary waves.

\section{Appendix: Tools}

5 All the tools presented above are available as FORTRAN code and UNIX/Linux shell scripts and can be obtained from the authors (fm265@cam.ac.uk).

Acknowledgements. We would like to thank M. Klawa for technical support and C. Schnadt for helpful discussions. This work was supported by the Bundesministerium für Bildung und Forschung (07ATF43).

\section{References}

Austin, J.: A three-dimensional coupled chemistry-climate model simulation of past stratospheric trends, J. Atmos. Sci., 59, 218-232, 2002. 2561

Austin, J., Shindell, D., Beagley, S. R., Brühl, C., Dameris, M., Manzini, E., Nagashima, T., Newman, P., Pawson, S., Pitari, G., Rozanov, E., Schnadt, C., and Shepherd, T. G.: Uncertainties and assessments of chemistry-climate models of the stratosphere, Atmos. Chem. Phys., 3, 1-27, 2003, SRef-ID: 1680-7324/acp/2003-3-1. 2561, 2570

Charney, J. G. and Drazin, P. G.: Propagation of planetary-scale disturbances from the lower into the upper atmosphere, J. Geophys. Res., 66, 83-109, 1961. 2568, 2572

Farrell, B.: The initial growth of disturbances in a baroclinic flow, J. Atmos. Sci., 39, 1663-1686, 1982. 2575

Gates, W. L.: AMIP: The atmospheric model intercomparison project, Bull. Amer. Meteor. Soc., 73, 1962-1970, 1992. 2570

Gibson, J. K., Kallberg, P., Uppala, S., Hernandez, A., Nomura, A., and Serrano, E.: ERA

5, 2559-2598, 2005

Planetary waves in model and reanalysis

F. Mager and M. Dameris

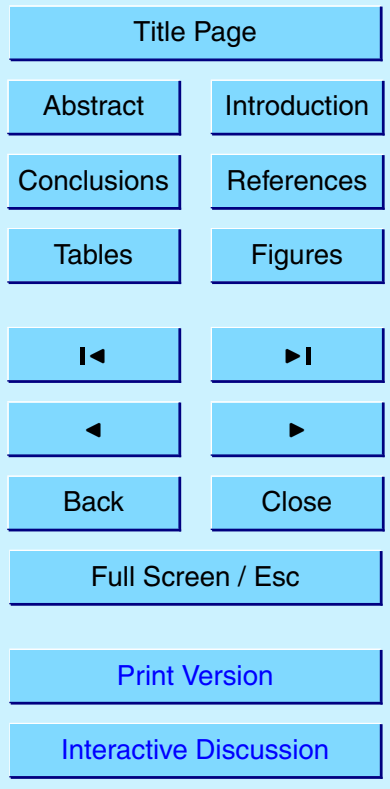

EGU 
Harnik, N. and Lindzen, R. S.: The effect of reflecting surfaces on the vertical structure and variability of stratospheric planetary waves, J. Atmos. Sci., 58, 2872-2894, 2001. 2569, 2582

Hartmann, D. L.: Baroclinic instability of realistic zonal mean states to planetary waves, J. Atmos. Sci., 36, 2336-2349, 1979. 2580

Hartmann, D. L.: Global Physical Climatology, Academic Press, International Geophysics Series, 56, 411, 1994. 2567

Hayashi, Y.: On the coherence between progressive and retrogressive waves and a partition of space-time power-spectra into standing parts, J. Meteor. Soc. Japan, 16, 368-373, 1977. $10 \quad 2562,2563,2565$

Hayashi, Y.: A generalized method of resolving transient disturbances into standing and traveling waves by space-time spectral analysis, J. Atmos. Sci., 36, 1017-1029, 1979. 2589

Hayashi, Y.: Space-time spectral analysis and its applications to atmospheric waves, J. Meteor. Soc. Japan, 60, 156-171, 1982. 2562, 2563, 2565

Hayashi, Y. and Golder, D. G.: Space-time spectral analysis of mid-latitude disturbances appearing in a GFDL general circulation model, J. Atmos. Sci., 34(2), 237-262, 1977. 2562

Hayashi, Y. and Golder, D. G.: Transient planetary waves simulated by GFDL spectral general circulation models. Part 1: Effects of montains, J. Atmos. Sci., 40(4), 941-950, 1983a. 2562

Hayashi, Y. and Golder, D. G.: Transient planetary waves simulated by GFDL spectral general circulation models. Part 2: Effects of nonlinear energy transfer, J. Atmos. Sci., 40(4), 951957, 1983b. 2562

Hayashi, Y. and Golder, D. G.: Kelvin and mixed Rossby-gravity waves appearing in the GFDL "SKYHI" general circulation model and the FGGE dataset: Implications for their generation mechanismn and role in the QBO, J. Meteorol. Soc. Japan, 72(6), 901-935, 1994. 2562

Hayashi, Y., Golder, D. G., and Jones P. W.: Tropical gravity waves and superclusters simulated by high-horizontal-resolution SKYHI general circulation models, J. Meteorol. Soc. Japan, 75(6), 1125-1139, 1997. 2562

Hein, R., Dameris, M., Schnadt, C., Land, C., Grewe, V., Köhler, I., Ponater, M., Sausen, R., Steil, B., Landgraf, J., and Brühl, C.: Results of an interactively coupled atmospheric chemistry-general circulation model: Comparison with observations, Ann. Geophys., 19, 435-457, 2001,

SRef-ID: 1432-0576/ag/2001-19-435. 2560, 2562, 2570, 2573, 2579, 2582

IPCC (Intergovernmental Panel on Climate Change): Climate change 2001; the scientific basis,

ACPD

5, 2559-2598, 2005

\section{Planetary waves in model and reanalysis}

F. Mager and M. Dameris

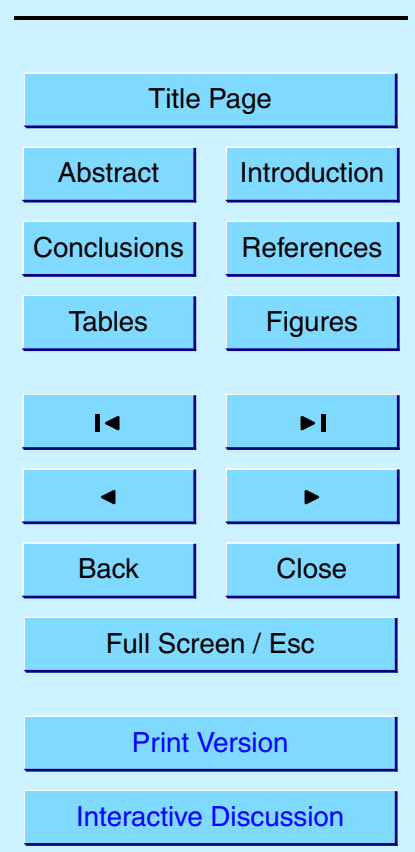

EGU 
contribution of working group I to the Third Assessment Report of IPCC, edited by: Houghton, J. T., Ding, Y., Griggs, D. J., Noguer, M., van der Linden, P. J. Dai, X., Maskell, K., and Johnson, C. A., Cambridge University Press, 2001. 2561

Jakobs, H. J. and Hass, H.: Normal modes as simulated in a three-dimensional circulation

5 model of the middle atmosphere including regional gravity wave activity, Ann. Geophys., 5A, 103-114, 1987. 2562

Kasahara, A.: Normal modes of ultralong waves in the atmosphere, Mon. Wea. Rev., 104, 669-690, 1976. 2577

Land, C., Feichter, J., and Sausen, R.: Impact of vertical resolution on the transport of passive tracers in the ECHAM4 model, Tellus, 54B, 344-360, 2002. 2570

Lindzen, R. S.: Baroclinic neutrality and the tropopause, J. Atmos. Sci., 50, 1148-1151, 1993. 2576

Madden, R.: Observations of large-scale traveling Rossby waves, Rev. Geophys. Space Phys., 17, 1935-1949, 1979. 2577

15 Matsuno, T.: Vertical propagation of stationary planetary waves in the winter Northern Hemisphere, J. Atmos. Sci., 27, 871-888, 1970. 2568

Miyoshi, Y.: Numerical simulation of the 5-day and 16-day waves in the mesopause region, Earth Planets Space, 51, 763-772, 1999. 2562, 2577

Miyoshi, Y. and Hirooka, T.: A numerical experiment of excitation of the 5-day wave by a GCM,

$20 \quad$ J. Atmos. Sci., 56, 1698-1707, 1999. 2562, 2577

Nagashima, T., Takahashi, M., Takigawa, M., and Akiyoshi, H.: Future development of the ozone layer calculated by a general circulation model with fully interactive chemistry, Geophys. Res. Lett., 29(8), 1162, doi:10.1029/2001GL014026, 2002. 2561

Newman, P. A. and Nash, E. R.: Quantifying the wave driving of the stratosphere, J. Geophys. Res., 105, 12 485-12 497, 2000. 2561, 2569

Peixoto, J. P. and Oort, A. H.: Physics of climate, Springer Verlag, 61-64, 1992. 2569

Perlwitz, J. and Harnik, N.: Observational evidence of a stratospheric influence on the troposphere by planetary wave reflection, J. Climate, 16, 3011-3026, 2003. 2569, 2582

Pitari, G., Mancini, E., Rizi, V., and Shindell, D.: Impact of future climate and emission changes on stratospheric aerosols and ozone, J. Atmos. Sci., 59, 414-440, 2002. 2561

Randel, W. J.: The seasonal evolution of planetary waves in the Southern Hemisphere stratosphere and troposphere, Quart. J. Roy. Meteorol. Soc., 114, 1385-1409, 1988. 2580

Rozanov, E. V., Schlesinger, M. E., and Zubov, V. A.: The Universtiy of Illinois at Urbana-

ACPD

5, 2559-2598, 2005

\section{Planetary waves in model and reanalysis}

F. Mager and M. Dameris

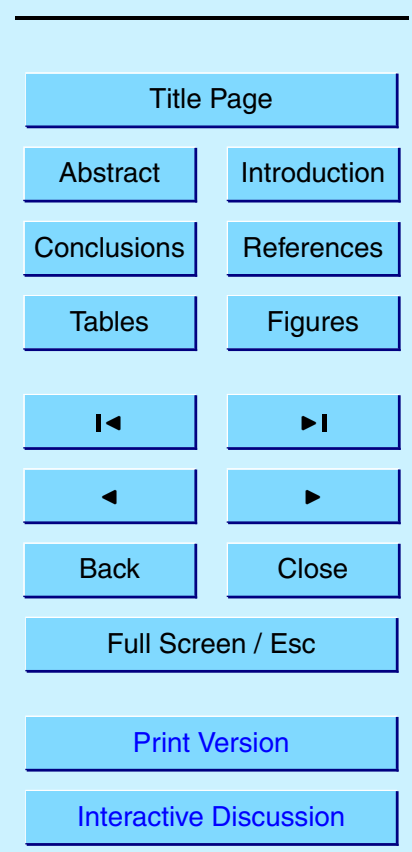

EGU 
Champaign three-dimensional stratosphere-troposphere general circulation model with interactive ozone photochemistry: Fifteen-year control run climatology, J. Geophys. Res., 106, 27 233-27 254, 2001. 2561

Salby, M. L.: A ubiquitous wavenumber- 5 anomaly in the southern hemisphere during FGGE, Mon. Wea. Rev., 110, 1712-1720, 1982. 2568

Santer, B. D., Sausen, R., Wigley, T. M. L., Boyle, J. S., AchutaRao, K., Doutriaux, C., Hansen, J. E., Meehl, G. A., Roeckner, E., Ruedy, R., Schmidt, G., and Taylor, K. E.: Behavior of tropopause height and atmospheric temperature in models, reanalyses, and observations: Decadal changes, J. Geophys. Res., 108(D1), 4002, doi:10.1029/2002JD002258, 2003. $10 \quad 2576$

Shepherd, T. G., Semeniuk, K., and Koshyk, J. N.: Sponge layer feedbacks in middle atmosphere models, J. Geophys. Res., 101, 23 447-23 464, 1996. 2579

Schnadt, C., Dameris, M., Ponater, M., Hein, R., Grewe, V., and Steil, B.: Interaction of atmospheric chemistry and climate and its impact on stratospheric ozone, Clim. Dyn., 18,

Senior, C. A.: The dependence of climate sensitivity on the horizontal resolution of a GCM, J. Climate, 8, 2860-2880, 1995. 2573

Shine, K. P., Bourqui, M. S., Forster, P. M. de F., Hare, S. H. E., Langematz, U., Braesicke, P., Grewe, V., Ponater, M., Schnadt, C., Smith, C. A., Haigh, J. D., Austin, J., Butchart, N., Shindell, D. T., Randel, W. J., Nagashima, T., Portmann, R. W., Solomon, S., Seidel, D. J., Lanzante, J., Klein, S., Ramaswamy, V., and Schwarzkopf, M. D.: A comparison of model-simulated trends in stratospheric temperatures, Q. J. R. Meteorol. Soc., 129(590), 1565-1588, 2003. 2561

Speth, P. and Kirk, E.: A one-year study of power spectra in wavenumber-frequency domain, Beitr. Phys. Atmosph., 54, 186-206, 1981. 2562

Speth, P. and Madden, R. A.: Space-time spectral analyses of northern hemisphere geopotential heights, J. Atmos. Sci., 40, 1086-1100, 1983. 2562, 2567

Steil, B., Dameris, M., Brühl, C., Crutzen, P. J., Grewe, V., Ponater, M., and Sausen, R.: Development of a chemistry module for GCMs: first results of a multiannual integration, Ann. 30 Geophys., 16, 205-228, 1998, SRef-ID: 1432-0576/ag/1998-16-205. 2570

Steil, B., Brühl, C., Manzini, E., Crutzen, P. J., Lelieveld, J., Rasch, P. J., Roeckner, E., and Krüger, K.: A new interactive chemistry climate model. 1: Present day climatology and inter-
ACPD

5, 2559-2598, 2005

\section{Planetary waves in model and reanalysis}

F. Mager and M. Dameris

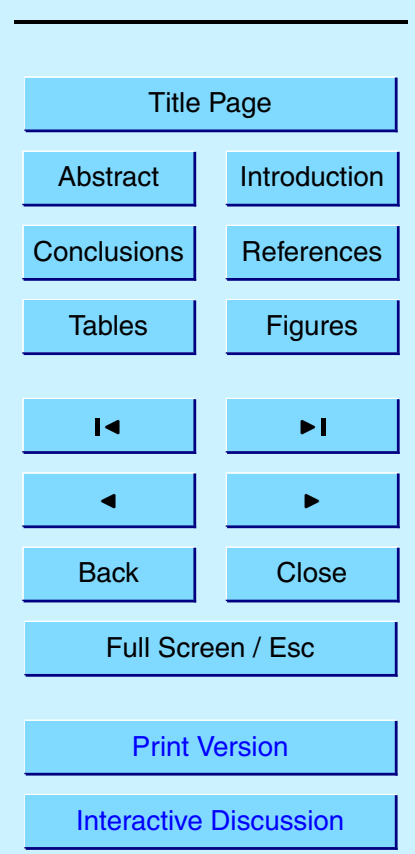

EGU 
annual variability of the middle atmosphere using the model and 9 years of HALOE/UARS data, J. Geophys. Res., 108(D9), 4290, doi:10.1029/2002JD002971, 2003. 2561

Zangvil, A.: On the presentation and interpretation of spectra of large-scale disturbances, Mon.

Wea. Rev., 105, 1469-1473, 1977. 2592

\section{ACPD}

$5,2559-2598,2005$

Planetary waves in model and reanalysis

F. Mager and M. Dameris

Title Page

Abstract

Introduction

Conclusions

References

Tables

Figures

14

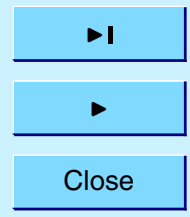

Full Screen / Esc

Print Version

Interactive Discussion

EGU 


\section{ACPD}

$5,2559-2598,2005$

Planetary waves in model and reanalysis

F. Mager and M. Dameris

Table 1. Mixing ratios of greenhouse gases, inorganic chlorine, and $\mathrm{NO}_{\mathrm{x}}$ emissions of different natural and anthropogenic sources for the "1990" simulation.

\begin{tabular}{lc}
\hline $\mathrm{CO}_{2}$ (ppmv) & 353 \\
$\mathrm{CH}_{4}$ (ppmv) & 1.69 \\
$\mathrm{~N}_{2} \mathrm{O}$ (ppbv) & 310 \\
$\mathrm{Cl}_{\mathrm{y}}$ (ppbv) & 3.4 \\
$\mathrm{NO}_{x}$ lightning $(\mathrm{Tg}(\mathrm{N}) /$ year) & 5.3 \\
$\mathrm{NO}_{\mathrm{x}}$ air traffic $(\mathrm{Tg}(\mathrm{N}) /$ year) & 0.6 \\
$\mathrm{NO}_{\mathrm{x}}$ surface (total) $(\mathrm{Tg}(\mathrm{N}) /$ year) & 33.1 \\
$\mathrm{NO}_{x}$ surface (industry, traffic) & 22.6 \\
$\mathrm{NO}_{\mathrm{x}}$ surface (soils) & 5.5 \\
$\mathrm{NO}_{x}$ surface (biomass burning) & 5.0
\end{tabular}

Title Page
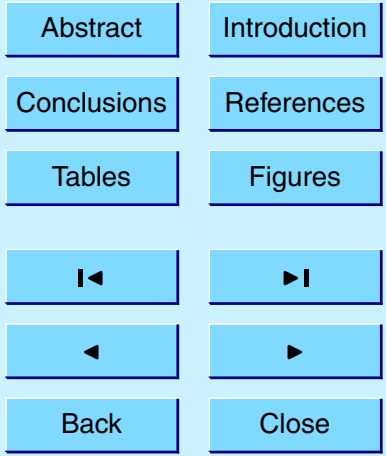

Full Screen / Esc

Print Version

Interactive Discussion

EGU 


\section{ACPD}

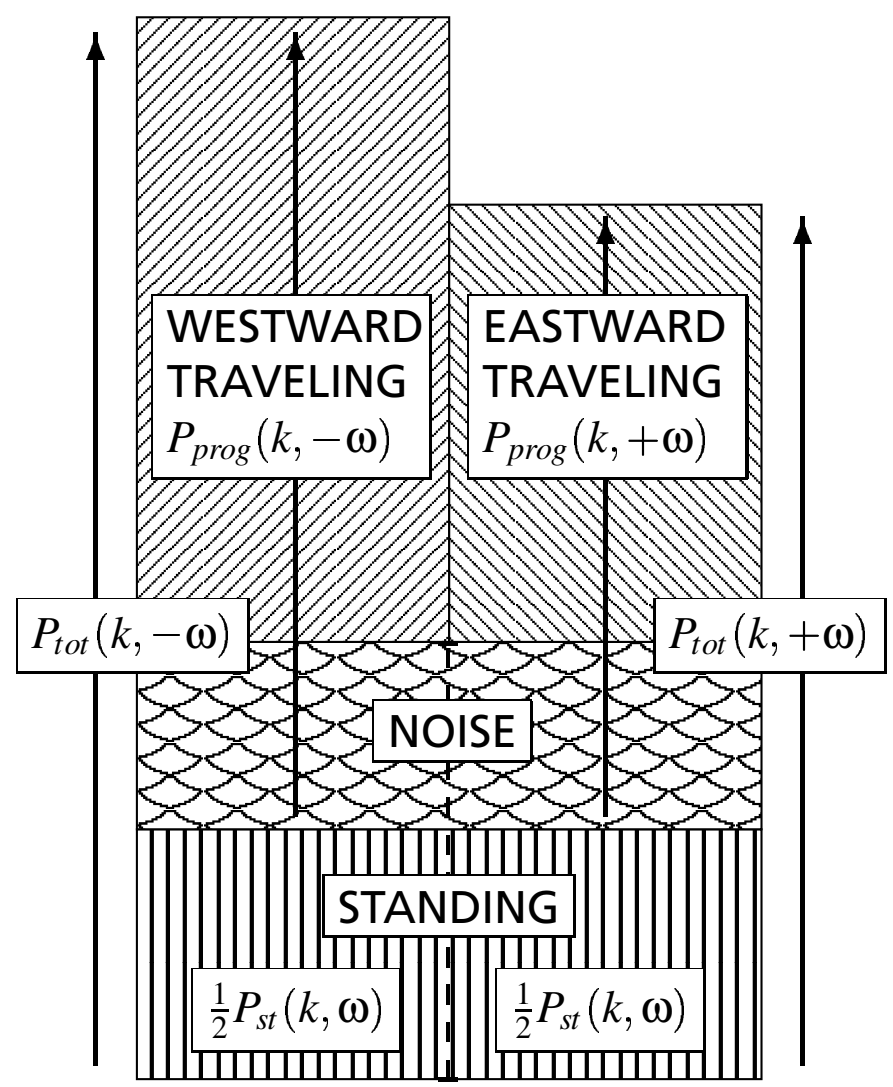

$5,2559-2598,2005$

Planetary waves in model and reanalysis

F. Mager and M. Dameris

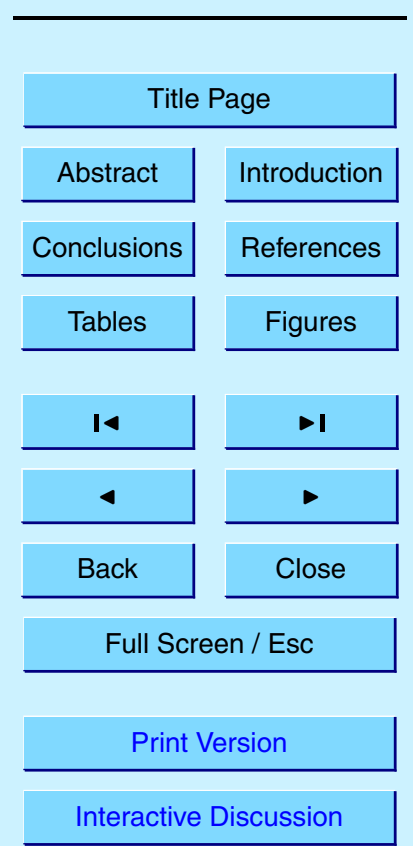

Fig. 1. Decomposition of standing and travelling wave parts after Hayashi (1979). The variance of progressive waves is obtained by substracting the standing parts from the total variance.

EGU 


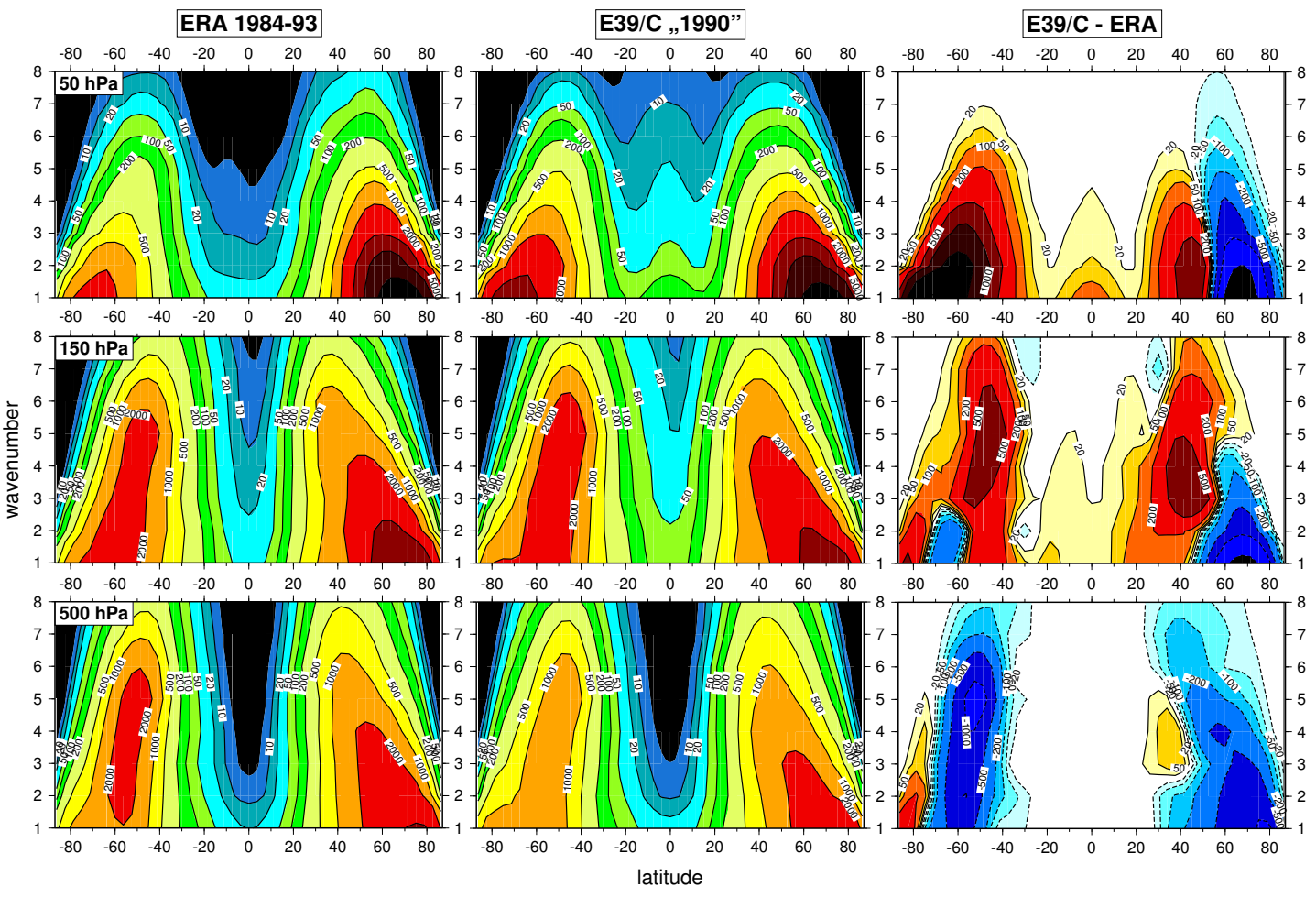

Fig. 2. Variance of geopotential height in $\mathrm{gpm}^{2}$ at 500,150 and $50 \mathrm{hPa}$ as computed by the WFA for DJF (Eqs. 8 and 9). Shown is the variance sum of standing and eastward and westward travelling waves as sum over 23 frequency bands with periods from 2.7 to 32 days, averaged over 10 winters for ERA data (left) and 20 winters for E39/C data (center). The right panels show the difference between ERA and E39/C variances; note that colours and contour values are different from those in left and centre panels. Isolines at 5, 10, 20, 50, 100, 200, 500, 1000, $2000,5000,10000$ and 20000 resp. $\pm 20,50,1000,200,500,1000$ and $2000 \mathrm{gpm}^{2}$.
ACPD

$5,2559-2598,2005$

Planetary waves in model and reanalysis

F. Mager and M. Dameris

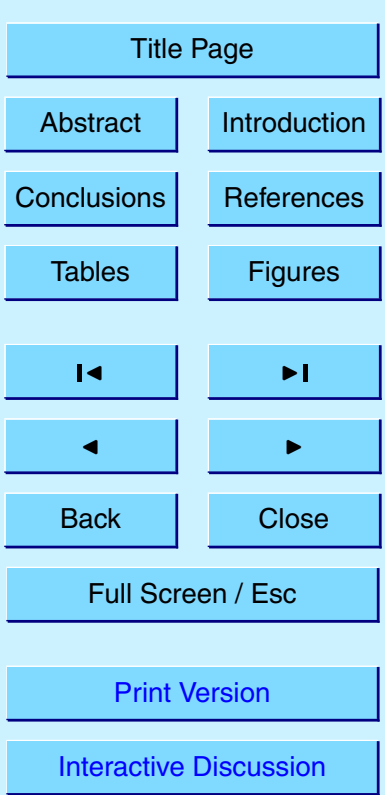

EGU 


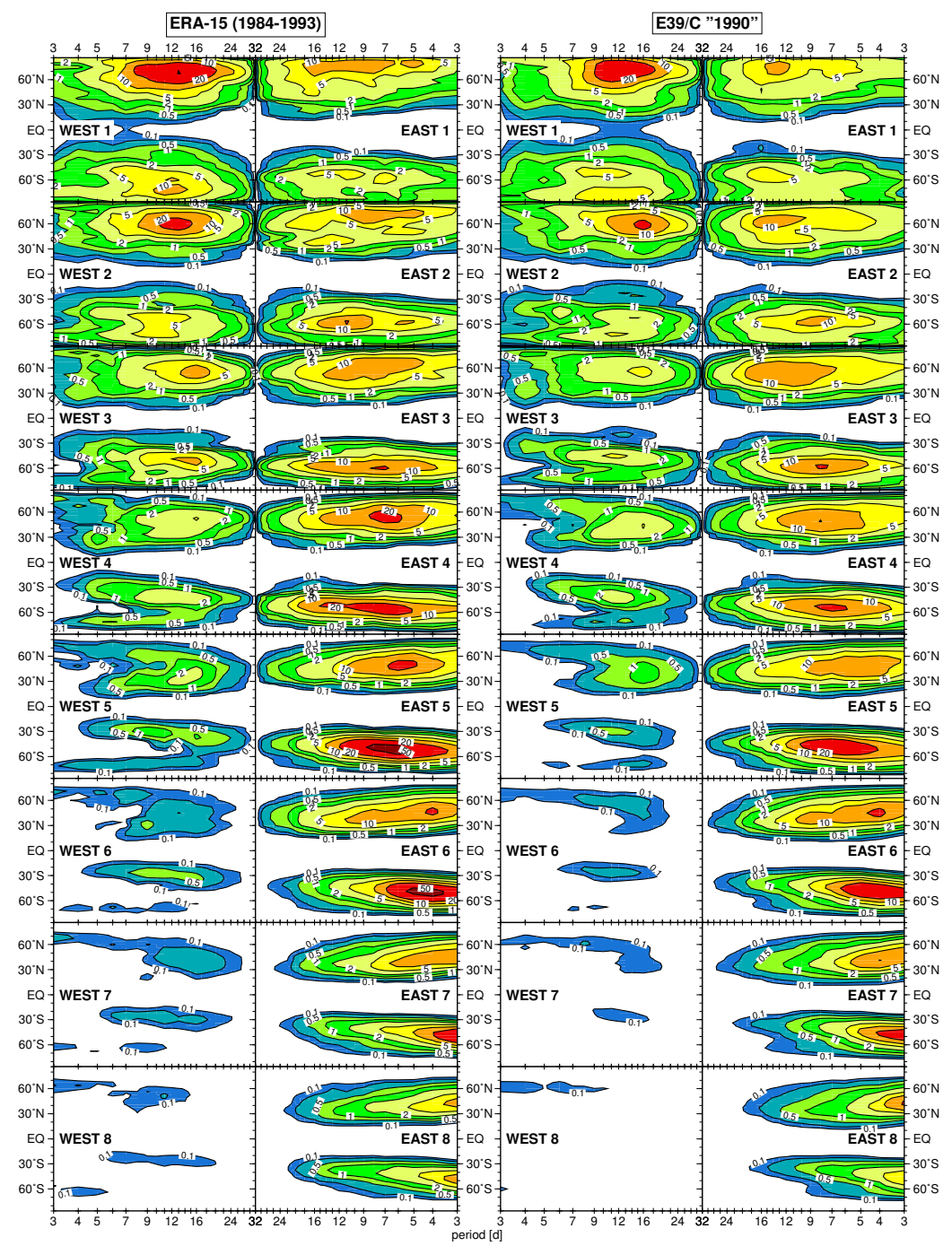

ACPD

5, 2559-2598, 2005

Planetary waves in model and reanalysis

F. Mager and M. Dameris

Title Page

\begin{tabular}{c|c|} 
Abstract & Introduction \\
\cline { 1 - 1 } Conclusions & References \\
\cline { 1 - 2 } &
\end{tabular}

Tables

Figures

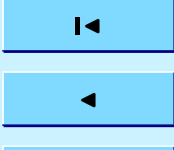

I

Back

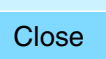

Full Screen / Esc

Print Version

Interactive Discussion

EGU 


\section{ACPD}

$5,2559-2598,2005$

\section{Planetary waves in} model and reanalysis

F. Mager and M. Dameris

Fig. 3. DJF variance of geopotential height per day at $300 \mathrm{hPa}$ for east- and westward travelling waves of wavenumbers 1-8 (top to bottom), for ERA-15 (10-year mean) and E39/C (20-year mean) as computed by the WFA. Variances have been multiplied by the corresponding frequency and plotted logarithmically over frequency so as to allow an area-conserving representation which respects the correct position of the dominant scale (Zangvil, 1977). Isolines at 0.1, $0.2,0.5,1,2,5,10,20$ and $50 \mathrm{gpm}^{2} \mathrm{~d}^{-1}$.

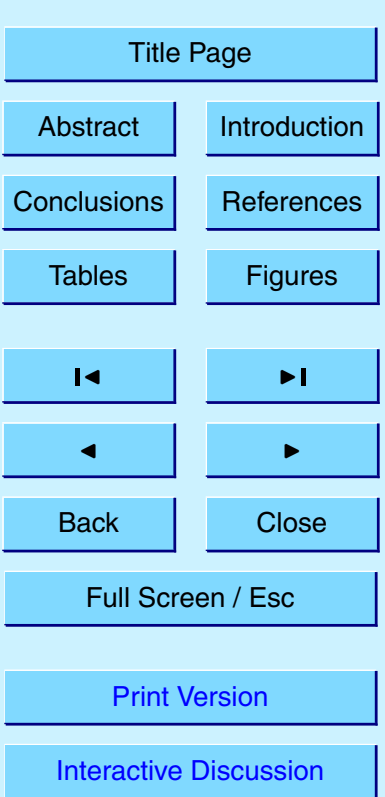

EGU 


\section{ACPD}

$5,2559-2598,2005$
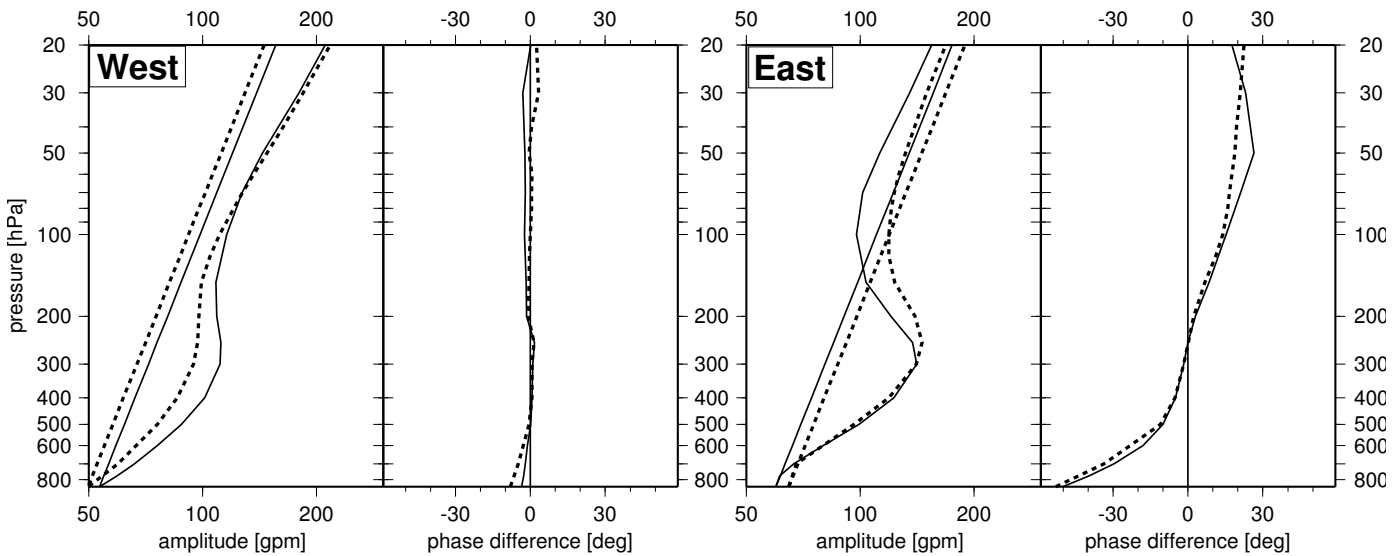

Planetary waves in model and reanalysis

F. Mager and M. Dameris

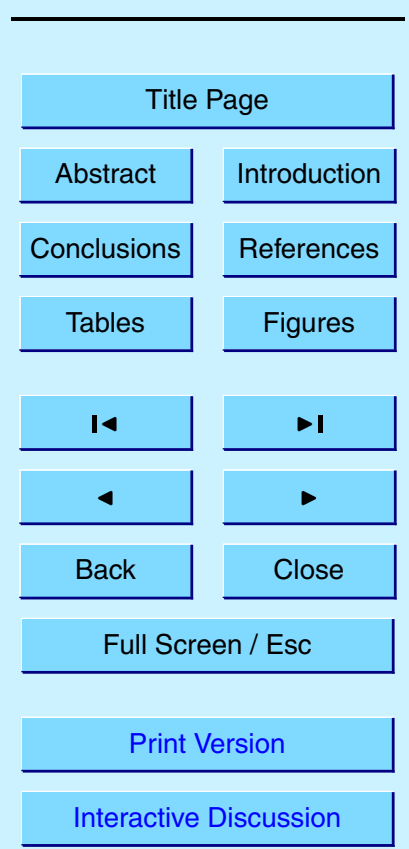

Fig. 4. Amplitudes [gpm] and phase differences [deg] of west- and eastward travelling disturbances of the geopotential height as computed by the WFA, for ERA (solid) and E39/C (dashed), as sum (amplitude) resp. mean (phase) over the first eight wavenumbers and all considered period intervals. The wave amplitude is computed from the square root of the doubled and previously summed variance. The vertical growth of the Lamb mode from the initial amplitude at $850 \mathrm{hPa}$ is seen as a straight line. Phase differences relative to $250 \mathrm{hPa}$ are given by Eq. (13). The respective latitude has been chosen to correspond to the $\mathrm{NH}$ meridional variance maximum $\left(70^{\circ} \mathrm{N}\right.$ for WEST, $50^{\circ} \mathrm{N}$ for EAST). 


\section{ACPD}

$5,2559-2598,2005$

Planetary waves in model and reanalysis

F. Mager and M. Dameris
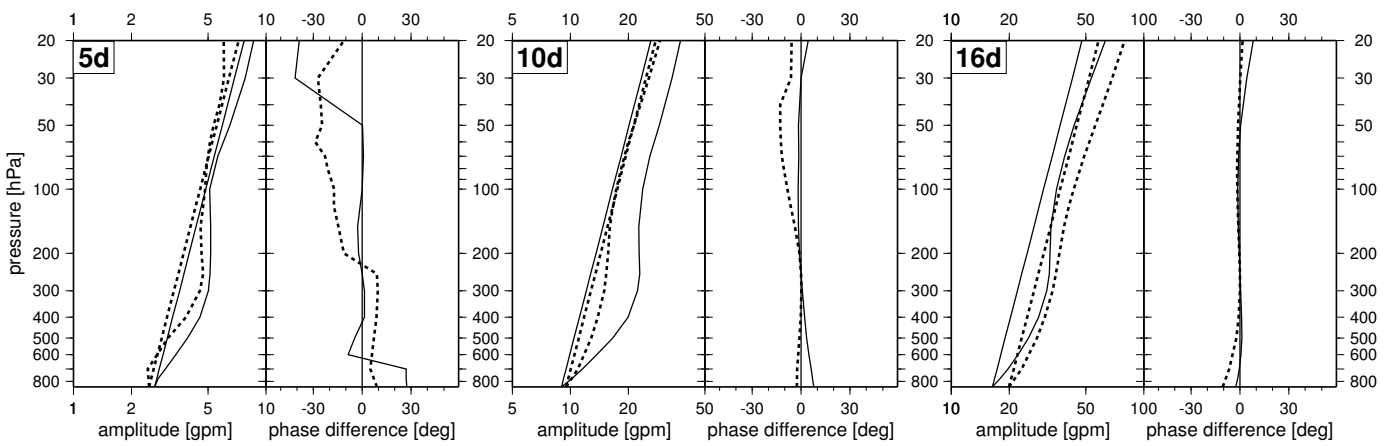

Fig. 5. Amplitudes and phase differences of geopotential height at $70^{\circ} \mathrm{N}$ from ERA (solid) and E39/C (dashed) for "normal modes" as computed by the WFA. These modes correspond to westward travelling waves of wavenumber 1 with approximate periods of 5,10 and 16 days.

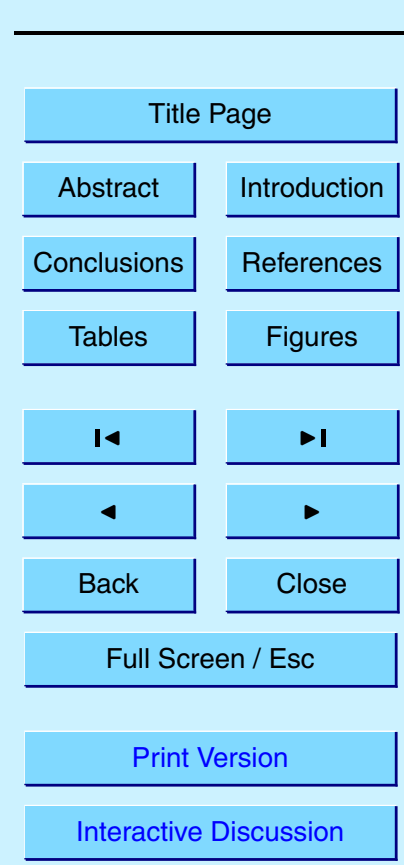

EGU 


\section{ACPD}

$5,2559-2598,2005$
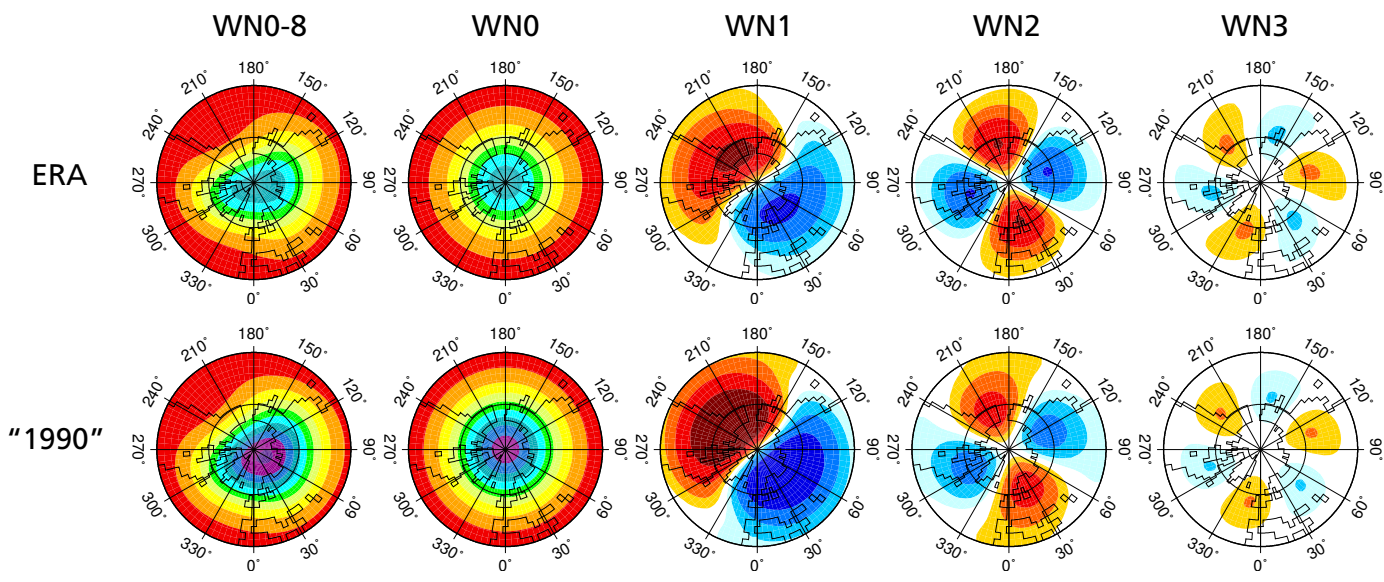

[gpkm]

18.618 .819 .019 .219 .419 .619 .820 .020 .220 .420 .6

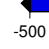

$-200$

Fig. 6. Geopotential height amplitudes of stationary waves at $50 \mathrm{hPa}$, derived for DJF from ERA (top, 10 year mean) and E39/C (bottom, 20 year mean) data, in polar stereographic projection from $30^{\circ} \mathrm{N}$ to $90^{\circ} \mathrm{N}$. Wavenumbers 0 to 3 are directly computed from space-Fourier coefficients whereas WN0-8 denotes the sum of the geopotential height zonal mean (WNO) and wavenumbers 1 to 8 .

Planetary waves in model and reanalysis

F. Mager and M. Dameris

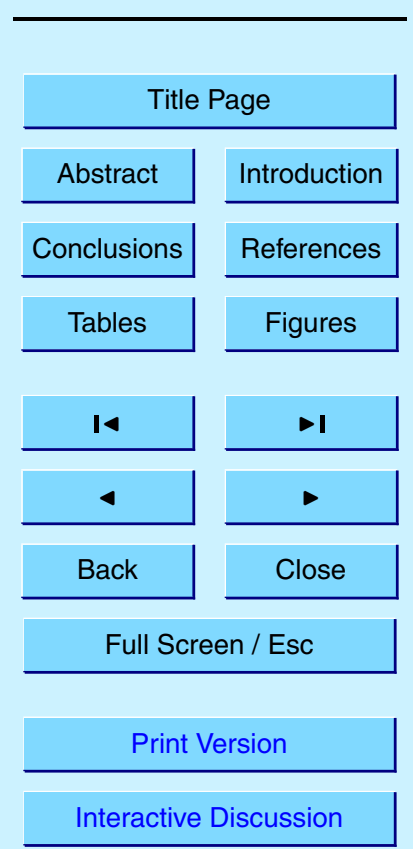

EGU 
ACPD

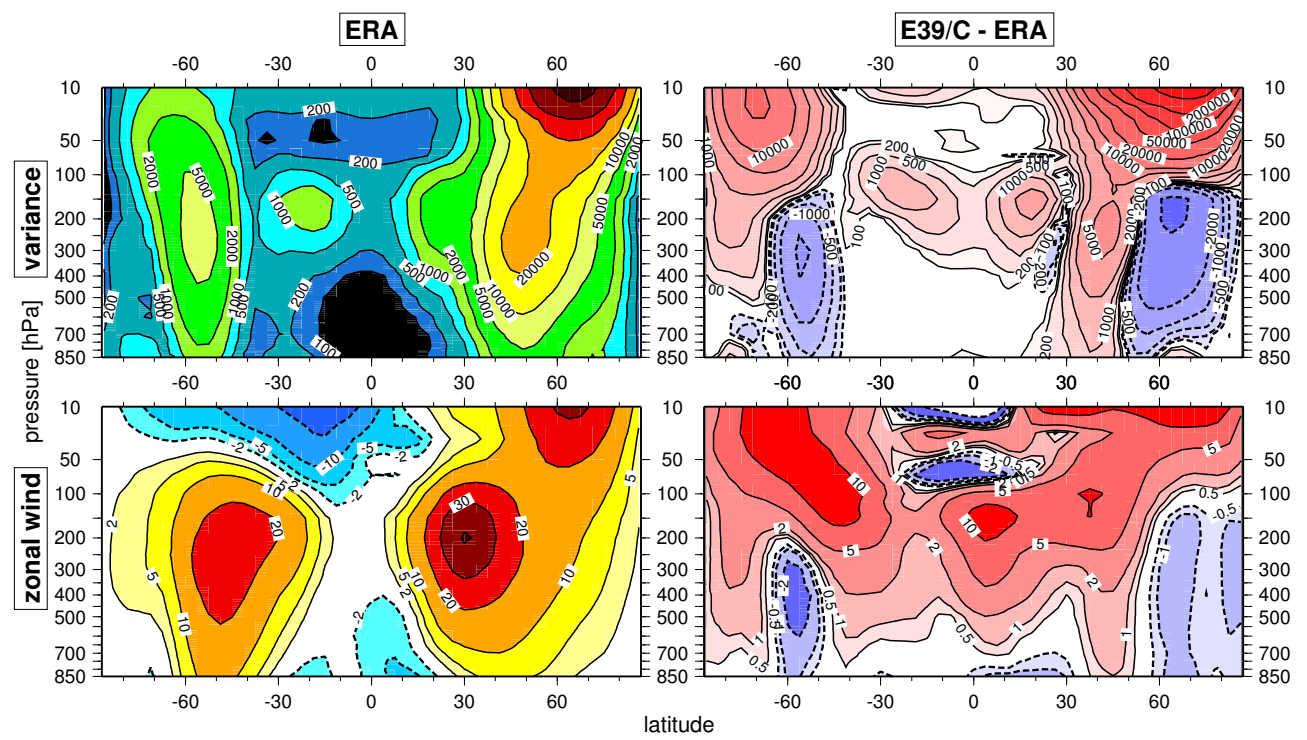

Fig. 7. Variance sum of stationary waves over wavenumbers 1-8 (top) and zonal mean of the zonal wind (bottom) for DJF. Top left: variance sum for ERA, isolines at 100, 200, 500, $1000,2000,5000,10000,20000,50000,1 \cdot 10^{5}$, and $2 \cdot 10^{5} \mathrm{gpm}^{2}$. Top right: variance sum as difference between model and reanalysis (E39/C-ERA), red colors indicate higher model variances, isolines at $\pm 100,200,500,1000,2000,5000,10000,20000,50000,1 \cdot 10^{5}, 2 \cdot 10^{5}$, and $5 \cdot 10^{5} \mathrm{gpm}^{2}$. Bottom left: zonal wind for ERA, dotted areas denote easterlies, isolines at $-20,-10,-5,-2,2,5,10,20,30$, and $40 \mathrm{~ms}^{-1}$. Bottom right: zonal wind as difference "E39/CERA", dotted areas denote lower model windspeeds, isolines at $-2,-1,-0.5,0.5,1,2,5$, and $10 \mathrm{~ms}^{-1}$.
$5,2559-2598,2005$

Planetary waves in model and reanalysis

F. Mager and M. Dameris

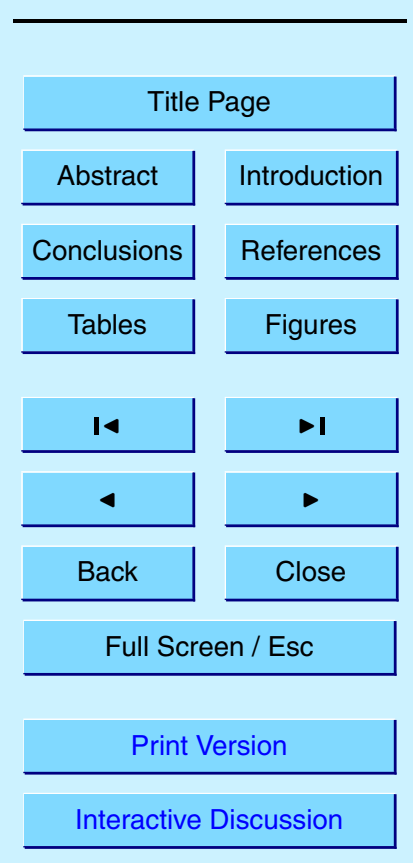

EGU 


\section{ACPD}

$5,2559-2598,2005$

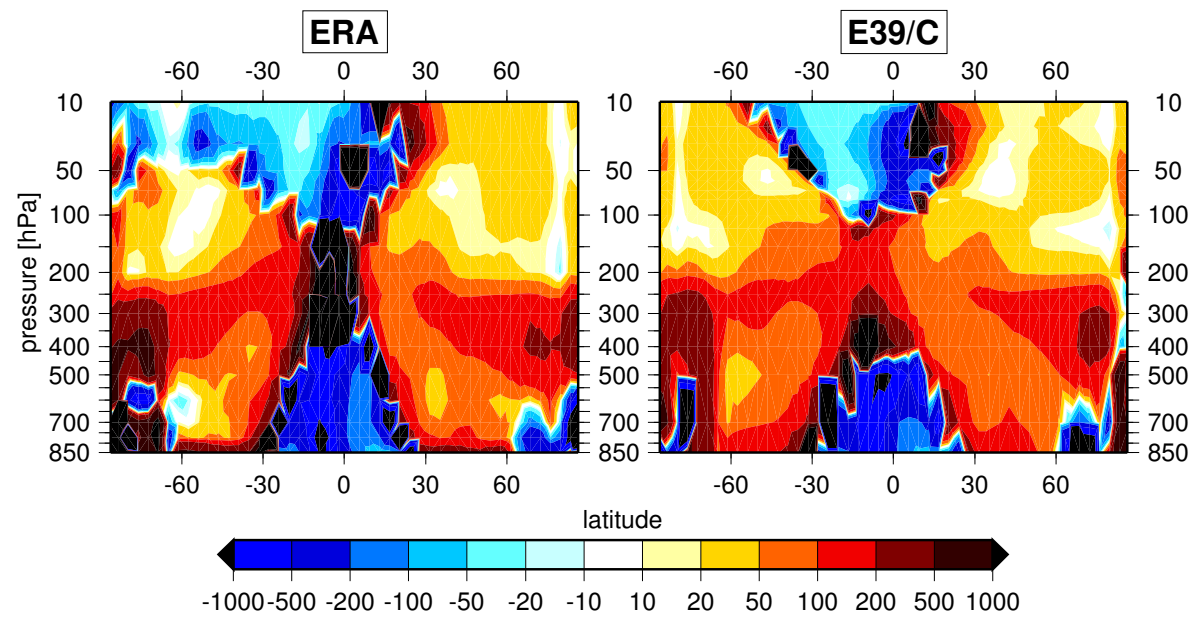

Fig. 8. Refractive index for wavenumber 1 as computed by Eq. (17). Positive values characterise regions where stationary waves can propagate vertically.

\section{Planetary waves in} model and reanalysis

F. Mager and M. Dameris

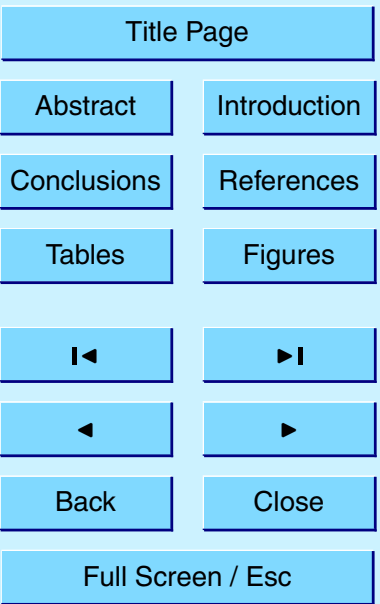

Print Version

Interactive Discussion

EGU 


\section{ACPD}

$5,2559-2598,2005$

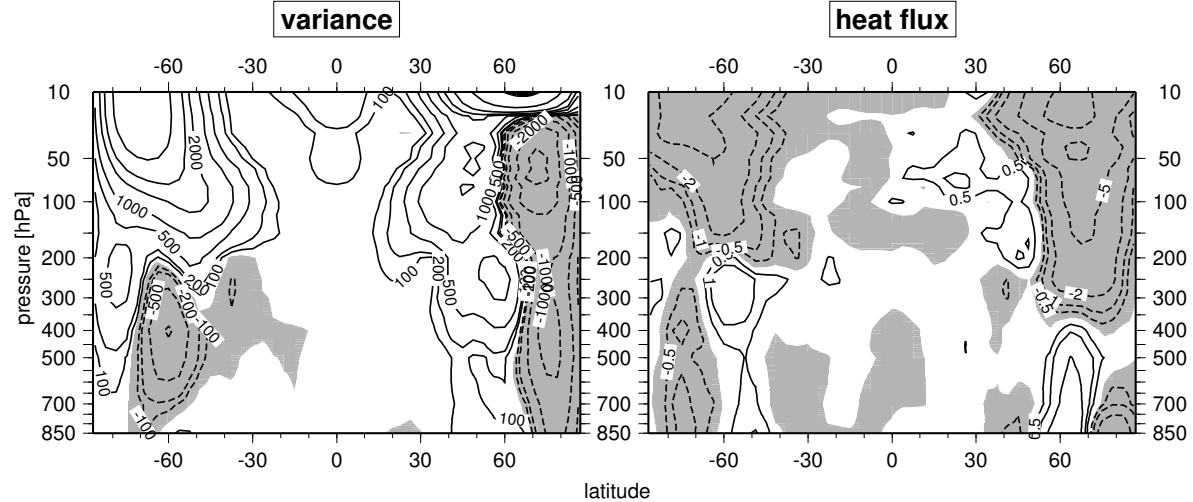

Fig. 9. "E39/C"-"ERA" differences of the variance of geopotential height (left, isolines at \pm 50 , $100,200,500,1000,2000,5000$ and $10000 \mathrm{gpm}^{2}$ ) and heat flux (right, isolines at $\pm 0.5,1,2$, 5 , and $10 \mathrm{~K}$ ) by transient waves with wavenumbers $1-3$. The variance is calculated by the WFA for eastward traveling waves and summed up over all considered periods. Negative variance and heat flux differences are shaded.
Planetary waves in model and reanalysis

F. Mager and M. Dameris

Title Page

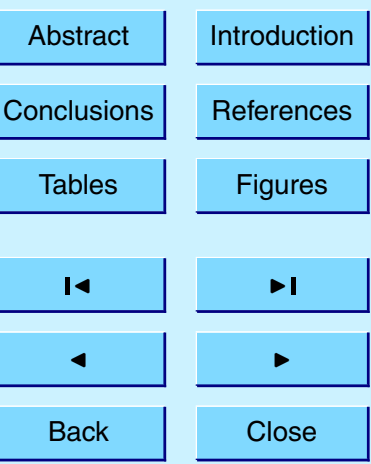

Full Screen / Esc

Print Version

Interactive Discussion

EGU 